\title{
Around Widder's characterization of the Laplace transform of an element of $L^{\infty}\left(\mathbb{R}^{+}\right)$
}

\author{
by JAN KISYŃSKI (Lublin)
}

Bogdan Ziemian in memoriam

\begin{abstract}
Let $\varkappa$ be a positive, continuous, submultiplicative function on $\mathbb{R}^{+}$such that $\lim _{t \rightarrow \infty} e^{-\omega t} t^{-\alpha} \varkappa(t)=a$ for some $\omega \in \mathbb{R}, \alpha \in \overline{\mathbb{R}^{+}}$and $a \in \mathbb{R}^{+}$. For every $\lambda \in(\omega, \infty)$ let $\phi_{\lambda}(t)=e^{-\lambda t}$ for $t \in \mathbb{R}^{+}$. Let $L_{\varkappa}^{1}\left(\mathbb{R}^{+}\right)$be the space of functions Lebesgue integrable on $\mathbb{R}^{+}$with weight $\varkappa$, and let $E$ be a Banach space. Consider the map $\phi_{\bullet}:(\omega, \infty) \ni \lambda \rightarrow$ $\phi_{\lambda} \in L_{\varkappa}^{1}\left(\mathbb{R}^{+}\right)$. Theorem 5.1 of the present paper characterizes the range of the linear map $T \rightarrow T \phi \bullet$ defined on $L\left(L_{\varkappa}^{1}\left(\mathbb{R}^{+}\right) ; E\right)$, generalizing a result established by B. Hennig and F. Neubrander for $\varkappa(t)=e^{\omega t}$. If $\varkappa \equiv 1$ and $E=\mathbb{R}$ then Theorem 5.1 reduces to D. V. Widder's characterization of the Laplace transform of a function in $L^{\infty}\left(\mathbb{R}^{+}\right)$. Some applications of Theorem 5.1 to the theory of one-parameter semigroups of operators are discussed. In particular a version of the Hille-Yosida generation theorem is deduced for $C_{0}$ semigroups $\left(S_{t}\right)_{t \in \mathbb{R}^{+}}$such that $\sup _{t \in \mathbb{R}^{+}}(\varkappa(t))^{-1}\left\|S_{t}\right\|<\infty$.
\end{abstract}

0. Introduction. Inversion formulas for the Laplace transform play an important role in the theory of one-parameter semigroups of operators. E. Hille's original proof of the Hille-Yosida generation theorem is related to the Post-Widder real formula for the inverse Laplace transform. This formula is close to

(a) Widder's "general representation theorem", [W;I], p. 303, and to

(b) Widder's characterization of the Laplace transform of an element of $L^{\infty}\left(\mathbb{R}^{+}\right)$, [W;I], pp. 315-316.

2000 Mathematics Subject Classification: Primary 44A10, 46G10, 47D06; Secondary 43A20, 46H15, 46B22.

Key words and phrases: real inversion formulas for the Laplace transform, operators from $L_{\varkappa}^{1}\left(\mathbb{R}^{+}\right)$into a Banach space, vector measures, complete monotonicity and positivity with respect to a cone, Radon-Nikodym property, Gelfand space, representations of the convolution algebra $L_{\varkappa}^{1}\left(\mathbb{R}^{+}\right)$, pseudoresolvents and their generators, one-parameter semigroups of operators. 
The generalization of (b) to functions with values in a Banach space, due to $\mathrm{W}$. Arendt $[\mathrm{A}]$, is important for the theory of integrated and regularized semigroups of operators. Arendt's proof of his generalization of (b) consists in a reduction to the classical Widder version of (b) by means of linear functionals. B. Hennig and F. Neubrander $[\mathrm{H}-\mathrm{N}]$ and A. Bobrowski $[B]$ have reproved Arendt's result in the framework of linear operators from $L^{1}\left(\mathbb{R}^{+}\right)$ into a Banach space. The argument in $[\mathrm{H}-\mathrm{N}]$ is based on (a). The proof in $[\mathrm{B}]$ is based on a "representation theorem" related to the R. S. Phillips real inversion formula for the Laplace transform ([Ph]; $[\mathrm{H}-\mathrm{P}]$, p. 221, Theorem 6.6.3).

Similarly to the above-mentioned result of $[\mathrm{H}-\mathrm{N}]$ and $[\mathrm{B}]$, Theorem 5.1 of the present paper (described in the Abstract) is a generalization of (b) from elements $T$ of $L^{\infty}\left(\mathbb{R}^{+}\right)$, i.e. linear functionals $T$ on $L^{1}\left(\mathbb{R}^{+}\right)$, to linear operators $T$ from $L_{\varkappa}^{1}\left(\mathbb{R}^{+}\right)$into a Banach space $E$. In contrast to [H-N], and similarly to [B], the proof of Theorem 5.1 is direct: neither (a) nor (b) is used in this proof, and (b) follows at once from Theorem 5.1 applied to $\varkappa \equiv 1$ and $E=\mathbb{R}$ or $E=\mathbb{C}$. Moreover, some estimations which are troublesome for general $\varkappa$, become obvious when $\varkappa \equiv 1$, and the proof of Theorem 5.1 for $\varkappa \equiv 1$ is simpler than Widder's original proof of (b), based on (a).

The space $L_{\varkappa}^{1}\left(\mathbb{R}^{+}\right)$is a convolution Banach algebra. Let $X$ be a Banach space, $L(X)$ the Banach algebra of continuous linear operators from $X$ into $X$, and $\operatorname{Hom}\left(L_{\varkappa}^{1}\left(\mathbb{R}^{+}\right) ; L(X)\right)$ the corresponding set of homomorphisms of Banach algebras. The elements of $\operatorname{Hom}\left(L_{\varkappa}^{1}\left(\mathbb{R}^{+}\right) ; L(X)\right)$ are nothing but continuous representations of the convolution Banach algebra $L_{\varkappa}^{1}\left(\mathbb{R}^{+}\right)$on the Banach space $X$. The present paper expounds the case when the operator $T$ occurring in Theorem 5.1 belongs to $\operatorname{Hom}\left(L_{\varkappa}^{1}\left(\mathbb{R}^{+}\right) ; L(X)\right)$. In this connection notice that if $T \in \operatorname{Hom}\left(L_{\varkappa}^{1}\left(\mathbb{R}^{+}\right) ; L(X)\right)$ and $D_{0}=\left\{\varphi \in C_{\mathrm{c}}^{\infty}(\mathbb{R})\right.$ : $\left.\operatorname{supp} \varphi \subset \overline{\mathbb{R}^{+}}\right\}$then the following two conditions are equivalent $\left(^{1}\right)$ :

(i) $\left.T\right|_{D_{0}}$ is an $L(X)$-valued semigroup distribution in the sense of J. L. Lions $[\mathrm{L}]$,

(ii) there is a unique $C_{0}$ semigroup $\left(S_{t}\right)_{t \in \overline{\mathbb{R}^{+}}} \subset L(X)$ such that $\sup _{t \in \overline{\mathbb{R}^{+}}}(\varkappa(t))^{-1}\left\|S_{t}\right\|<\infty$ and

$$
T(\varphi) x=\int_{0}^{\infty} \varphi(t) S_{t} x d t
$$

$\left({ }^{1}\right)$ Indeed, (ii) $\Rightarrow$ (i) is an easy consequence of Definition 1.1 in $[L]$, p. 142 . On the other hand, by (4.1) and (9.3) in the present paper, $T \phi \bullet \in W_{\varkappa}(L(X))$ and $T \phi \bullet$ is a pseudoresolvent with values in $L(X)$. By Lemma 2.1 and Proposition 11.4 of the present paper, if (i) holds, then conditions (iv) and (v) of Definition $1.1 \mathrm{in}[\mathrm{L}]$ imply that $T \phi \bullet$ is the resolvent of a densely defined, closed linear operator $A$ from $X$ into $X$. By Theorem 12.2 and Lemma 2.1, $A$ is the infinitesimal generator of a semigroup satisfying (ii). 
for all $\varphi \in L_{\varkappa}^{1}\left(\mathbb{R}^{+}\right)$and $x \in X$, the integral being understood in the sense of Bochner $\left({ }^{2}\right)$.

Theorem 12.2 of the present paper deals with $C_{0}$ operator semigroups and refines the Hille-Yosida generation theorem. For every $M \in[1, \infty)$ and every function $\varkappa$ with properties as in the abstract, Theorem 12.2 characterizes the class of the infinitesimal generators of $C_{0}$ semigroups $\left(S_{t}\right)_{t \in \overline{\mathbb{R}^{+}}}$such that $\sup _{t \in \overline{\mathbb{R}^{+}}}(\varkappa(t))^{-1}\left\|S_{t}\right\|=M$, while the Hille-Yosida theorem gives such a characterization only for exponential functions $\varkappa$. The proof is based on the reconstruction of a semigroup from (a candidate for) the infinitesimal generator along the scheme: the generator $\stackrel{(1)}{\rightarrow}$ the resolvent $\stackrel{(2)}{\rightarrow}$ the homomorphism of the convolution algebra $L_{\varkappa}^{1}\left(\mathbb{R}^{+}\right)$into the Banach algebra $L(X) \stackrel{(3)}{\rightarrow}$ the semigroup. Step (1) is standard. Step (2) is based on Theorem 5.1 which contains all the technical difficulties. Step (3) resembles a procedure known in the representation theory of groups.

Acknowledgements. The author is greatly indebted to Wojciech Chojnacki for helpful discussions, for drawing the author's attention to the factorization theorem for Banach algebras, and for indicating the analogues of Theorem 10.2 in the theory of representations of groups.

1. The weight function $\varkappa$ and the space $L_{\varkappa}^{1}\left(\mathbb{R}^{+}\right)$. Throughout this paper $\mathbb{R}^{+}=(0, \infty), \overline{\mathbb{R}^{+}}=[0, \infty)$, the numbers $\omega \in \mathbb{R}, \alpha \in \overline{\mathbb{R}^{+}}$and $a \in \mathbb{R}^{+}$ are fixed, and $\varkappa$ is a fixed strictly positive, continuous, submultiplicative function on $\overline{\mathbb{R}^{+}}$such that $\varkappa(0)=1$ and

$$
\lim _{t \rightarrow \infty} \frac{\varkappa(t)}{e^{\omega t} t^{\alpha}}=a .
$$

Submultiplicativity of $\varkappa$ means that

$$
\varkappa(t+u) \leq \varkappa(t) \varkappa(u) \quad \text { for all } t \text { and } u \text { in } \overline{\mathbb{R}^{+}} .
$$

Let $K$ be either $\mathbb{R}$ or $\mathbb{C}$, and let $L_{\varkappa}^{1}\left(\mathbb{R}^{+}\right)$be the space of the equivalence classes of $K$-valued functions Lebesgue integrable on $\mathbb{R}^{+}$with weight $\varkappa$. Equipped with the norm

$$
\|\varphi\|_{1, \varkappa}=\int_{0}^{\infty}|\varphi(t)| \varkappa(t) d t, \quad \varphi \in L_{\varkappa}^{1}\left(\mathbb{R}^{+}\right),
$$

$L_{\varkappa}^{1}\left(\mathbb{R}^{+}\right)$is a Banach space.

$\left({ }^{2}\right)$ Representations of $L^{1}\left(\mathbb{R}^{+}\right)$defined in this way are (implicitly) used in the proof given in $[\mathrm{S}-\mathrm{K}]$, pp. 307-309, of the Trotter-Kato approximation theorem for $C_{0}$ semigroups. 
The strict positivity, continuity and the property (1.1) of $\varkappa$ imply that

$$
\left.\lim _{\lambda \rightarrow \infty}\left(\varkappa_{0}(t)\right)^{-1} \frac{\lambda^{n+1}}{n !} \int_{0}^{\infty} s^{n} e^{-\lambda s} \varkappa_{0}(s) d s\right|_{n=[\lambda t]}=1
$$

uniformly with respect to $t \in \overline{\mathbb{R}^{+}}$, where

$$
\varkappa_{0}(t)=e^{-\omega t} \varkappa(t) \text {. }
$$

The uniformity in (1.2) is crucial for the proof of Theorem 5.1. The pointwise limit (1.2) is considered in $[\mathrm{W} ; \mathrm{I}]$, p. 289, Theorem $6 \mathrm{~b}$, under assumptions weaker than ours. The standard weight functions used in the theory of operator semigroups have the form $\varkappa(t)=e^{\omega t}$, so that $\varkappa_{0} \equiv 1$ and the uniformity in (1.2) is a trivial consequence of the equality

$$
\frac{\lambda^{n+1}}{n !} \int_{0}^{\infty} s^{n} e^{-\lambda s} d s=1, \quad \lambda \in \mathbb{R}^{+} .
$$

However in some papers concerning integrated operator semigroups (for instance $\left[\right.$ deL-H-W-W], [deL-J]) the weight functions $\varkappa(t)=e^{\omega t}(1+c t)^{k}$ with $\omega \in \mathbb{R}, c \in \mathbb{R}^{+}, k=0,1, \ldots$ are considered. It is natural to associate such weight functions with semigroups of $(k+1) \times(k+1)$ matrices of the form

$$
\begin{aligned}
& \exp \left(t\left(\begin{array}{ccccc}
\omega & c & & & \\
& \omega & c & & \\
& & \ddots & \ddots & \\
& & & \omega & c \\
& & & & \omega
\end{array}\right)\right) \\
&=e^{\omega t}\left(\begin{array}{ccccc}
1 & c t & (c t)^{2} / 2 & \ldots & (c t)^{k} / k ! \\
& 1 & c t & \ldots & (c t)^{k-1} /(k-1) ! \\
& & & \ddots & \\
& & & & c t
\end{array}\right) .
\end{aligned}
$$

Except for the proof of the uniformity in (1.2), the use of our more general weight functions does not cause any complications. Conditions (1.1) and (1.2) are insignificant in Sections 2, 3, 8 and 10, and in Sections 2-10 and 12 it is natural to use as general weight functions as possible.

In the proof of the uniformity in (1.2) we use the equality

$$
\lim _{x \rightarrow \infty} \frac{\Gamma(x+\alpha)}{x^{\alpha} \Gamma(x)}=1
$$

for the Euler $\Gamma$ function, which is an easy consequence of the Stirling formula ([R;I], Sec. 8.22). For all $\mu \in \mathbb{R}^{+}$and $\beta \in \mathbb{R}^{+}$consider the probabilistic gamma density on $\overline{\mathbb{R}^{+}}([\mathrm{J}-\mathrm{K}-\mathrm{B}]$, Chap. 17$)$ 


$$
\gamma_{\lambda, \beta}(s)=\frac{\lambda^{\beta}}{\Gamma(\beta)} s^{\beta-1} e^{-\lambda s}, \quad s \in \overline{\mathbb{R}^{+}}
$$

The mean value of $\gamma_{\lambda, \beta}$ is $\beta / \lambda$, and the variance is $\beta / \lambda^{2}$. A standard application of the Chebyshev inequality shows that

$$
\text { if } \varphi \text { is a bounded uniformly continuous function on } \overline{\mathbb{R}^{+}} \text {, then }
$$

$$
\lim _{\lambda \rightarrow \infty} \int_{0}^{\infty} \gamma_{\lambda,[\lambda t]+1}(s) \varphi(s) d s=\varphi(t)
$$

uniformly with respect to $t \in \overline{\mathbb{R}^{+}}$.

In terms of the densities $\gamma_{\lambda, \beta}$ the equality (1.2) takes the form

$$
\lim _{\lambda \rightarrow \infty}\left(\varkappa_{0}(t)\right)^{-1} \int_{0}^{\infty} \gamma_{\lambda,[\lambda t]+1}(s) \varkappa_{0}(s) d s=1 .
$$

Lemma 1.1. The limit relation (1.5) holds uniformly with respect to $t$ in any compact subinterval of $\overline{\mathbb{R}^{+}}$.

Proof. Fix any $t_{0} \in \mathbb{R}^{+}$. We will prove that (1.5) holds uniformly with respect to $t \in\left[0, t_{0}\right]$. To this end take any $T \in\left(t_{0}+1, \infty\right)$ and define

$$
c_{T}(s)= \begin{cases}1 & \text { if } s \in[0, T-1], \\ T-s & \text { if } s \in(T-1, T), \\ 0 & \text { if } s \in[T, \infty) .\end{cases}
$$

From (1.4) it follows that

$$
\lim _{\lambda \rightarrow \infty}\left(\varkappa_{0}(t)\right)^{-1} \int_{0}^{\infty} \gamma_{\lambda,[\lambda t]+1}(s) c_{T}(s) \varkappa_{0}(s) d s=1
$$

uniformly with respect to $t \in[0, T-1]$. Hence the proof of Lemma 1.1 will be complete once it is shown that

$$
\lim _{\lambda \rightarrow \infty} \sup _{t \in\left[0, t_{0}\right]} \int_{T-1}^{\infty} \gamma_{\lambda,[\lambda t]+1}(s) \varkappa_{0}(s) d s=0 .
$$

Let $C=\sup _{s \in \overline{\mathbb{R}^{+}}} \varkappa_{0}(s) e^{-s}$. Then $C<\infty$, by (1.1). If $\lambda \in\left(T /\left(T-1-t_{0}\right), \infty\right)$ then, by Chebyshev's inequality,

$$
\begin{aligned}
& \sup _{t \in\left[0, t_{0}\right]} \int_{T-1}^{\infty} \gamma_{\lambda,[\lambda t]+1}(s) \varkappa_{0}(s) d s \\
& \leq C \sup _{t \in\left[0, t_{0}\right]}\left(\frac{\lambda}{\lambda-1}\right)^{[\lambda t]+1} \int_{T-1}^{\infty} \gamma_{\lambda-1,[\lambda t]+1}(s) d s
\end{aligned}
$$




$$
\begin{aligned}
& \leq C\left(1+\frac{1}{\lambda-1}\right)^{(\lambda-1) t_{0}+\left(t_{0}+1\right)} \sup _{t \in\left[0, t_{0}\right]}\left(T-1-\frac{[\lambda t]+1}{\lambda-1}\right)^{-2} \frac{[\lambda t]+1}{(\lambda-1)^{2}} \\
& \leq C e^{t_{0}}\left(1+\frac{1}{\lambda-1}\right)^{t_{0}+1} \frac{\lambda t_{0}+1}{\left(\lambda\left(T-1-t_{0}\right)-T\right)^{2}},
\end{aligned}
$$

whence (1.7) follows.

Proposition 1.2. The limit relation (1.2) holds uniformly with respect to $t \in \overline{\mathbb{R}^{+}}$.

Pr o of. For every $T \in[1, \infty)$ define

$$
\begin{array}{rlrl}
m_{T} & =\inf _{s \geq T-1} \frac{\varkappa_{0}(s)}{s^{\alpha}}, & M_{T}=\sup _{s \geq T-1} \frac{\varkappa_{0}(s)}{s^{\alpha}}, \\
n_{T} & =\inf _{t \geq T} \frac{t^{\alpha}}{\varkappa_{0}(t)}, & N_{T} & =\sup _{t \geq T} \frac{t^{\alpha}}{\varkappa_{0}(t)} .
\end{array}
$$

Condition (1.1) implies that $\lim _{T \rightarrow \infty} m_{T}=\lim _{T \rightarrow \infty} M_{T}=a, \lim _{T \rightarrow \infty} n_{T}=$ $\lim _{T \rightarrow \infty} N_{T}=a^{-1}$, and hence

$$
\lim _{T \rightarrow \infty} m_{T} n_{T}=\lim _{T \rightarrow \infty} M_{T} N_{T}=1 .
$$

For all $\lambda \in \mathbb{R}^{+}, t \in \overline{\mathbb{R}^{+}}$, and every continuous function $\varphi$ on $\overline{\mathbb{R}^{+}}$such that $\sup _{s \geq 1}|\varphi(s)| / s^{\alpha}<\infty$ define

$$
I_{\lambda, t}(\varphi)=\int_{0}^{\infty} \gamma_{\lambda,[\lambda t]+1}(s) \varphi(s) d s .
$$

Proposition 1.2 is equivalent to the claim that

$$
\lim _{\lambda \rightarrow \infty} \sup _{t \in \overline{\mathbb{R}^{+}}}\left|\left(\varkappa_{0}(t)\right)^{-1} I_{\lambda, t}\left(\varkappa_{0}\right)-1\right|=0 .
$$

By Lemma 1.1,

$$
\lim _{\lambda \rightarrow \infty} \sup _{t \in[0, T]}\left|\left(\varkappa_{0}(t)\right)^{-1} I_{\lambda, t}\left(\varkappa_{0}\right)-1\right|=0 \quad \text { for every } T \in \mathbb{R}^{+} .
$$

Hence, by (1.8), Proposition 1.2 will follow once it is shown that

$$
\begin{aligned}
m_{T} n_{T} & \leq \liminf _{\lambda \rightarrow \infty} \inf _{t \in[T, \infty)}\left(\varkappa_{0}(t)\right)^{-1} I_{\lambda, t}\left(\varkappa_{0}\right) \\
& \leq \limsup _{\lambda \rightarrow \infty} \sup _{t \in[T, \infty)}\left(\varkappa_{0}(t)\right)^{-1} I_{\lambda, t}\left(\varkappa_{0}\right) \leq M_{T} N_{T}
\end{aligned}
$$

for every $T \in[1, \infty)$.

To prove (1.9), take any $T \in[1, \infty)$ and define the function $c_{T}$ by (1.6). Then

$$
m_{T} s^{\alpha}-m_{T} c_{T}(s) s^{\alpha} \leq \varkappa_{0}(s) \leq M_{T} s^{\alpha}+c_{T}(s) \varkappa_{0}(s)
$$


for every $s \in \overline{\mathbb{R}^{+}}$. As a consequence,

$$
\begin{aligned}
& m_{T} n_{T} t^{-\alpha} I_{\lambda, t}\left(\varphi_{\alpha}\right)-m_{T} D I_{\lambda, t}\left(c_{T} \varphi_{\alpha}\right) \\
& \quad \leq\left(\varkappa_{0}(t)\right)^{-1} I_{\lambda, t}\left(\varkappa_{0}\right) \leq M_{T} N_{T} t^{-\alpha} I_{\lambda, t}\left(\varphi_{\alpha}\right)+D I_{\lambda, t}\left(c_{T} \varkappa_{0}\right)
\end{aligned}
$$

for every $t \in[T, \infty)$, where $D=\sup _{t \in \overline{\mathbb{R}}^{+}}\left(\varkappa_{0}(t)\right)^{-1}<\infty$, and

$$
\varphi_{\alpha}(s)=s^{\alpha} \quad \text { for } s \in \overline{\mathbb{R}^{+}} .
$$

Since the function $c_{T}$ vanishes on $[T, \infty)$, from (1.4) and (1.10) it follows that

$$
m_{T} n_{T} \liminf _{\lambda \rightarrow \infty} \inf _{t \in[T, \infty)} t^{-\alpha} I_{\lambda, t}\left(\varphi_{\alpha}\right) \leq \liminf _{\lambda \rightarrow \infty} \inf _{t \in[T, \infty)}\left(\varkappa_{0}(t)\right)^{-1} I_{\lambda, t}\left(\varkappa_{0}\right)
$$

and

$$
\limsup _{\lambda \rightarrow \infty} \sup _{t \in[T, \infty)}\left(\varkappa_{0}(t)\right)^{-1} I_{\lambda, t}\left(\varkappa_{0}\right) \leq M_{T} N_{T} \limsup _{\lambda \rightarrow \infty} \sup _{t \in[T, \infty)} t^{-\alpha} I_{\lambda, t}\left(\varphi_{\alpha}\right) .
$$

These inequalities reduce the proof of (1.9) to showing that

$$
\lim _{\lambda \rightarrow \infty} \sup _{t \in[T, \infty)}\left|t^{-\alpha} I_{\lambda, t}\left(\varphi_{\alpha}\right)-1\right|=0 .
$$

To this end, note that

$$
\begin{aligned}
t^{-\alpha} I_{\lambda, t}\left(\varphi_{\alpha}\right) & =\frac{\lambda^{[\lambda t]+1}}{t^{\alpha}[\lambda t] !} \int_{0}^{\infty} s^{[\lambda t]+\alpha} e^{-\lambda s} d s \\
& =\frac{\lambda^{[\lambda t]+1}}{t^{\alpha}[\lambda t] !} \cdot \frac{\Gamma([\lambda t]+1+\alpha)}{\lambda^{[\lambda t]+1+\alpha}} \\
& =\left(\frac{[\lambda t]+1}{\lambda t}\right)^{\alpha} \frac{\Gamma([\lambda t]+1+\alpha)}{([\lambda t]+1)^{\alpha} \Gamma([\lambda t]+1)} .
\end{aligned}
$$

Hence

$$
\begin{aligned}
\sup _{t \in[T, \infty)} & \left|t^{-\alpha} I_{\lambda, t}\left(\varphi_{\alpha}\right)-1\right| \\
& \leq\left(\left(1+\frac{1}{\lambda T}\right)^{\alpha}-1\right) \sup _{x \geq \lambda T} \frac{\Gamma(x+\alpha)}{x^{\alpha} \Gamma(x)}+\sup _{x \geq \lambda T}\left|\frac{\Gamma(x+\alpha)}{x^{\alpha} \Gamma(x)}-1\right| .
\end{aligned}
$$

By (1.3), the last estimate implies (1.11).

2. The exponentials in $L_{\varkappa}^{1}\left(\mathbb{R}^{+}\right)$. For every $\lambda \in(\omega, \infty)$ let $\phi_{\lambda}$ be the element of $L_{\varkappa}^{1}\left(\mathbb{R}^{+}\right)$represented by the exponential function $t \rightarrow e^{-\lambda t}$ restricted to $\mathbb{R}^{+}$. Since $(\partial / \partial \lambda)^{n} e^{-\lambda t}=(-t)^{n} e^{-\lambda t}$, it follows that the map

$$
\phi_{\bullet}:(\omega, \infty) \ni \lambda \rightarrow \phi_{\lambda} \in L_{\varkappa}^{1}\left(\mathbb{R}^{+}\right)
$$


is infinitely differentiable in the norm of $L_{\varkappa}^{1}\left(\mathbb{R}^{+}\right)$. In terms of the map $\phi \bullet$ the uniform limit condition (1.2) takes the form

$$
\left.\lim _{\lambda \rightarrow \infty}\left(\varkappa_{0}(t)\right)^{-1} \frac{\lambda^{n+1}}{n !}\left\|\left(\frac{\partial}{\partial \lambda}\right)^{n} \phi_{\lambda+\omega}\right\|_{1, \varkappa}\right|_{n=[\lambda t]}=1
$$

uniformly with respect to $t \in \overline{\mathbb{R}^{+}}$. If $t=0$ then $n=[\lambda t]=0$, and (2.2) yields

$$
\lim _{\lambda \rightarrow \infty} \lambda\left\|\phi_{\lambda}\right\|_{1, \varkappa}=1 .
$$

Lemma 2.1. The set $\left\{\phi_{\lambda}: \lambda \in(\omega, \infty)\right\}$ is linearly dense in $L_{\varkappa}^{1}\left(\mathbb{R}^{+}\right)$.

Proof. For every $u \in \mathbb{R}^{+}$let $1_{(0, u]}$ be the element of $L_{\varkappa}^{1}\left(\mathbb{R}^{+}\right)$represented by the characteristic function of the interval $(0, u]$. The set $\operatorname{span}_{K}\left\{1_{(0, u]}\right.$ : $\left.u \in \mathbb{R}^{+}\right\}$is dense in $L_{\varkappa}^{1}\left(\mathbb{R}^{+}\right)$because it consists of all those elements of $L_{\varkappa}^{1}\left(\mathbb{R}^{+}\right)$which are represented by $K$-valued piecewise constant functions on $\mathbb{R}^{+}$with bounded supports. Therefore it is sufficient to show that

$$
1_{(0, u]} \in \overline{\operatorname{span}}_{\mathbb{R}}\left\{\phi_{\lambda}: \lambda \in(\omega, \infty)\right\}
$$

for every $u \in \mathbb{R}^{+}$, where the closure is taken in the norm topology of $L_{\varkappa}^{1}\left(\mathbb{R}^{+}\right)$.

To prove (2.4), fix any $u \in \mathbb{R}^{+}$. By $(2.3)$, for every $\lambda \in(\omega, \infty)$ the series

$$
\sum_{n=1}^{\infty}(-1)^{n+1} \frac{e^{n \lambda u}}{n !} \phi_{n \lambda}
$$

of elements of $L_{\varkappa}^{1}\left(\mathbb{R}^{+}\right)$is absolutely convergent in the norm of $L_{\varkappa}^{1}\left(\mathbb{R}^{+}\right)$. The sum $\psi_{\lambda, u}$ of this series is in $\overline{\operatorname{span}}_{\mathbb{R}}\left\{\phi_{n \lambda}: n=1,2, \ldots\right\}$, and hence (2.4) will follow once we prove

$$
\lim _{\lambda \rightarrow \infty}\left\|\psi_{\lambda, u}-1_{(0, u]}\right\|_{1, \varkappa}=0 .
$$

The element $\psi_{\lambda, u}$ of $L_{\varkappa}^{1}\left(\mathbb{R}^{+}\right)$is represented by the function

$$
\psi_{\lambda, u}^{0}(t)=-\sum_{n=1}^{\infty} \frac{\left(-e^{\lambda(u-t)}\right)^{n}}{n !}=1-\exp \left(-e^{\lambda(u-t)}\right) .
$$

Thus $0 \leq \psi_{\lambda, u}^{0}(t) \leq 1$ and

$$
\psi_{\lambda, u}^{0}(t)=\left.e^{s}\right|_{s=-e^{\lambda(u-t)}} ^{s=0}<e^{\lambda(u-t)},
$$

whence

$$
\int_{u}^{\infty} \psi_{\lambda, u}^{0}(t) \varkappa(t) d t \leq \varkappa(u) \int_{u}^{\infty} e^{-\lambda(t-u)} \varkappa(t-u) d t=\varkappa(u)\left\|\phi_{\lambda}\right\|_{1, \varkappa},
$$


so that

$$
\begin{aligned}
\left\|\psi_{\lambda, u}-1_{(0, u]}\right\|_{1, \varkappa} & =\int_{0}^{u}\left(1-\psi_{\lambda, u}^{0}(t)\right) \varkappa(t) d t+\int_{u}^{\infty} \psi_{\lambda, u}^{0}(t) \varkappa(t) d t \\
& \leq \int_{0}^{u} \varkappa(t) \exp \left(-e^{\lambda(u-t)}\right) d t+\varkappa(u)\left\|\phi_{\lambda}\right\|_{1, \varkappa} .
\end{aligned}
$$

By the Lebesgue dominated convergence theorem, and by (2.3), the last estimate implies (2.5).

The above proof is inspired by a remark on p. 165 of $[\mathrm{H}-\mathrm{N}]$ concerning the Phragmén inversion formula for the Laplace-Stieltjes transform.

3. The spaces $L\left(L_{\varkappa}^{1}\left(\mathbb{R}^{+}\right) ; E\right), \operatorname{Lip}_{\varkappa}\left(\overline{\mathbb{R}^{+}} ; E\right)$ and $M_{\varkappa}(E)$. Let $E$ be a Banach space over the field $K$, and $L\left(L_{\varkappa}^{1}\left(\mathbb{R}^{+}\right) ; E\right)$ the space of continuous linear operators from $L_{\varkappa}^{1}\left(\mathbb{R}^{+} ; K\right)$ into $E$. Denote by $\operatorname{Lip}_{\varkappa}\left(\overline{\mathbb{R}^{+}} ; E\right)$ the set of $E$-valued functions $g$ on $\overline{\mathbb{R}^{+}}$satisfying the condition $g(0)=0$ and having finite norm

$$
\|g\|_{\operatorname{Lip}_{\varkappa}}=\sup _{0 \leq s<t<\infty}\left(\int_{s}^{t} \varkappa(u) d u\right)^{-1}\|g(t)-g(s)\|_{E} .
$$

Equipped with this norm, $\operatorname{Lip}_{\varkappa}\left(\overline{\mathbb{R}^{+}} ; E\right)$ is a Banach space.

Let $\mathfrak{R}$ be the ring of Lebesgue measurable subsets $B$ of $\mathbb{R}^{+}$such that $\int_{B} \varkappa(t) d t<\infty$. Define $M_{\varkappa}(E)$ to be the set of $E$-valued measures $G$ on $\mathfrak{R}\left({ }^{3}\right)$ such there is a finite positive $C$ satisfying

$$
\|G(B)\|_{E} \leq C \int_{B} \varkappa(t) d t \quad \text { for every } B \in \mathfrak{R} .
$$

Equipped with the norm

$$
\|G\|_{M_{\varkappa}}=\text { the smallest } C \text { satisfying (3.1), }
$$

$M_{\varkappa}(E)$ is a Banach space.

If $T \in L\left(L_{\varkappa}^{1}\left(\mathbb{R}^{+}\right) ; E\right)$ then

$$
G_{T}: \Re \ni B \rightarrow T 1_{B} \in E
$$

is a measure in $M_{\varkappa}(E)$ such that $\left\|G_{T}\right\|_{M_{\varkappa}} \leq\|T\|$, and

$$
g_{T}: \overline{\mathbb{R}^{+}} \ni t \rightarrow T 1_{(0, t]} \in E
$$

\footnotetext{
$\left({ }^{3}\right)$ The terminology used here follows [Ha], pp. 19, 24, 30. Estimate (3.1) implies that $\lim _{n \rightarrow \infty}\left\|G\left(B_{n}\right)\right\|_{E}=0$ whenever $\left(B_{n}\right)_{n \in \mathbb{N}}$ is a decreasing sequence of sets in $\mathfrak{R}$ such that $\bigcap_{n \in \mathbb{N}} B_{n}=\emptyset$. It follows that $G$ is countably additive on $\mathfrak{R}$, that is, $G\left(\bigcup_{n \in \mathbb{N}} B_{n}\right)=$ $\sum_{n \in \mathbb{N}} G\left(B_{n}\right)$ whenever $\left(B_{n}\right)_{n \in \mathbb{N}}$ is a disjoint sequence in $\mathfrak{R}$ such that $\bigcup_{n \in \mathbb{N}} B_{n} \in \mathfrak{R}$.
} 
is a function in $\operatorname{Lip}_{\varkappa}\left(\overline{\mathbb{R}^{+}} ; E\right)$ such that $\left\|g_{T}\right\|_{\operatorname{Lip}_{\varkappa}} \leq\|T\|$. If $G \in M_{\varkappa}(E)$ then

$$
g_{G}: \overline{\mathbb{R}^{+}} \ni t \rightarrow G((0, t]) \in E
$$

is a function in $\operatorname{Lip}_{\varkappa}\left(\overline{\mathbb{R}^{+}} ; E\right)$ such that $\left\|g_{G}\right\|_{\operatorname{Lip}_{\varkappa}} \leq\|G\|_{M_{\varkappa}}$.

Proposition 3.1. The map $I_{1}: T \rightarrow G_{T}$ is a linear isometry of $L\left(L_{\varkappa}^{1}\left(\mathbb{R}^{+}\right) ; E\right)$ onto $M_{\varkappa}(E)$. The map $I_{2}: T \rightarrow g_{T}$ is a linear isometry of $L\left(L_{\varkappa}^{1}\left(\mathbb{R}^{+}\right) ; E\right)$ onto $\operatorname{Lip}_{\varkappa}\left(\overline{\mathbb{R}^{+}} ; E\right)$. Hence the map $I_{2} \circ I_{1}^{-1}: G \rightarrow g_{G}$ is a linear isometry of $M_{\varkappa}(E)$ onto $\operatorname{Lip}_{\varkappa}\left(\overline{\mathbb{R}^{+}} ; E\right)$.

Proof. Since the maps are linear and contractive, it remains to prove that

for every $G \in M_{\varkappa}(E)$ there is a unique $T \in L\left(L_{\varkappa}^{1}\left(\mathbb{R}^{+}\right) ; E\right)$ such that $G_{T}=G$ and $\|T\| \leq\|G\|_{M_{\varkappa}}$,

and

(3.3) for every $g \in \operatorname{Lip}_{\varkappa}\left(\overline{\mathbb{R}^{+}} ; E\right)$ there is a unique $T \in L\left(L_{\varkappa}^{1}\left(\mathbb{R}^{+}\right) ; E\right)$ such that $g_{T}=g$ and $\|T\| \leq\|g\|_{\operatorname{Lip}_{\varkappa}}$.

To this end, let $S_{1}$ be the set of those elements $\varphi$ of $L_{\varkappa}^{1}\left(\mathbb{R}^{+}\right)$which are represented by functions $\varphi_{0}$ for which the set $\varphi_{0}\left(\mathbb{R}^{+}\right)$is finite. Then $\varphi_{0}^{-1}(c) \in \Re$ for every $c \in K \backslash\{0\}$. Let $S_{2}$ be the set of those elements $\varphi$ of $L_{\varkappa}^{1}\left(\mathbb{R}^{+}\right)$which are represented by piecewise constant functions $\varphi_{0}$. If $T \in L\left(L_{\varkappa}^{1}\left(\mathbb{R}^{+}\right) ; E\right), G=G_{T}$ and $g=g_{T}$ then

$$
T \varphi=\sum_{c \in \varphi_{0}\left(\mathbb{R}^{+}\right) \backslash\{0\}} c G\left(\varphi_{0}^{-1}(c)\right) \quad \text { for every } \varphi \in S_{1}
$$

and

$$
T \varphi=\sum_{c \in \varphi_{0}\left(\mathbb{R}^{+}\right) \backslash\{0\}} c\left[g\left(\sup \varphi_{0}^{-1}(c)\right)-g\left(\inf \varphi_{0}^{-1}(c)\right)\right] \quad \text { for every } \varphi \in S_{2},
$$

so that the restriction $\left.T\right|_{S_{1}}$ is uniquely determined by $G$, and $\left.T\right|_{S_{2}}$ is uniquely determined by $g$. Since $S_{1}$ and $S_{2}$ are dense subsets of $L_{\varkappa}^{1}\left(\mathbb{R}^{+}\right)$, the operator $T \in L\left(L_{\varkappa}^{1}\left(\mathbb{R}^{+}\right) ; E\right)$ is uniquely determined by each of the above restrictions, and the uniqueness parts of (3.2) and (3.3) follow.

To prove the existence part of (3.2), take any $G \in M_{\varkappa}(E)$ and define $T: S_{1} \rightarrow E$ by (3.4). Then

$$
\|T \varphi\|_{E} \leq \sum_{c \in \varphi_{0}\left(\mathbb{R}^{+}\right) \backslash\{0\}}|c| \cdot\|G\|_{M_{\varkappa}} \int_{\varphi_{0}^{-1}(c)} \varkappa(t) d t=\|G\|_{M_{\varkappa}} \cdot\|\varphi\|_{1, \varkappa}
$$

for every $\varphi \in S_{1}$. Since $S_{1}$ is dense in $L_{\varkappa}^{1}\left(\mathbb{R}^{+}\right)$, it follows that $T$ can be uniquely extended to an operator belonging to $L\left(L_{\varkappa}^{1}\left(\mathbb{R}^{+}\right) ; E\right)$. If the extension is denoted again by $T$, then $\|T\| \leq\|G\|_{M_{\varkappa}}$, and $T 1_{B}=G(B)$ for every $B \in \mathfrak{R}$, by (3.4). The existence part of (3.3) may be proved by a similar argument based on (3.5). 
If $T \in L\left(L_{\varkappa}^{1}\left(\mathbb{R}^{+}\right) ; E\right)$ then

$$
T \varphi=\int_{0}^{\infty} \varphi(t) G_{T}(d t) \quad \text { for every } \varphi \in L_{\varkappa}^{1}\left(\mathbb{R}^{+}\right),
$$

the integral with respect to the $E$-valued measure $G_{T}$ being understood in the Lebesgue-Bartle-Dunford-Schwartz sense ([D-S;I], Sec. IV.10; [D-U], p. 56). In particular,

$$
T \phi_{\lambda}=\int_{0}^{\infty} e^{-\lambda t} G_{T}(d t) \quad \text { for every } \lambda \in(\omega, \infty),
$$

so that the function $(\omega, \infty) \ni \lambda \rightarrow T \phi_{\lambda} \in E$ is the Laplace transform of the $E$-valued measure $G_{T}$. Since $g_{T}(t)=G_{T}((0, t])$, a formal integration by parts leads from (3.7) to

$$
T \phi_{\lambda}=\lambda \int_{0}^{\infty} e^{-\lambda t} g_{T}(t) d t \quad \text { for every } \lambda \in(\omega, \infty) .
$$

This formula is indeed true if the integral is understood in the Bochner sense. For the proof, observe that for every $\lambda \in(\omega, \infty)$ the continuous $L_{\varkappa}^{1}\left(\mathbb{R}^{+}\right)$-valued function $\mathbb{R}^{+} \ni t \rightarrow \lambda e^{-\lambda t} 1_{(0, t]} \in L_{\varkappa}^{1}\left(\mathbb{R}^{+}\right)$is absolutely integrable on $\mathbb{R}^{+}$and the Bochner integral $\int_{0}^{\infty} \lambda e^{-\lambda t} 1_{(0, t]} d t$ is an element of $L_{\varkappa}^{1}\left(\mathbb{R}^{+}\right)$represented by the function

$$
s \rightarrow \int_{0}^{\infty} \lambda e^{-\lambda t} 1_{(0, t]}(s) d t=\lambda \int_{s}^{\infty} e^{-\lambda t} d t=e^{-\lambda s} .
$$

Hence $\int_{0}^{\infty} \lambda e^{-\lambda t} 1_{(0, t]} d t=\phi_{\lambda}$, and so

$$
T \phi_{\lambda}=\int_{0}^{\infty} \lambda e^{-\lambda t} T 1_{(0, t]} d t=\lambda \int_{0}^{\infty} e^{-\lambda t} g_{T}(t) d t .
$$

4. The Widder space $W_{\varkappa}(E)$. If $E$ is a Banach space over the field $K$ then we define $W_{\varkappa}(E)$ as the normed linear space over $K$ whose elements are those maps $f:(\omega, \infty) \rightarrow E$ which are infinitely differentiable in the norm topology of $E$ and have finite norm

$$
\|f\|_{W_{\varkappa}(E)}=\sup \left\{\frac{\left\|(\partial / \partial \lambda)^{n} f(\lambda)\right\|_{E}}{\left\|(\partial / \partial \lambda)^{n} \phi_{\lambda}\right\|_{1, \varkappa}}: \lambda \in(\omega, \infty), n=0,1, \ldots\right\} .
$$

The term by term differentiation theorem implies that $W_{\varkappa}(E)$ is a Banach space. For reasons which will be explained after Corollary 5.2, we call $W_{\varkappa}(E)$ the Widder space. The map $\phi_{\bullet}$ defined by (2.1) belongs to $W_{\varkappa}\left(L_{\varkappa}^{1}\left(\mathbb{R}^{+}\right)\right)$and $\left\|\phi_{\bullet}\right\|_{W_{\varkappa}\left(L_{\varkappa}^{1}\left(\mathbb{R}^{+}\right)\right)}=1$. 
LEMMA 4.1. If $E$ is a Banach space and $f \in W_{\varkappa}(E)$, then the map $f:(\omega, \infty) \rightarrow E$ is real analytic and for every $\mu \in(\omega, \infty)$ the Taylor series of $f$ with center at $\mu$ has convergence radius no smaller than $\mu-\omega$.

Proof. Fix any $\mu \in(\omega, \infty)$ and $\lambda \in(\omega, 2 \mu-\omega)$. We have to prove that $\lim _{n \rightarrow \infty}\left\|R_{n}\right\|_{E}=0$, where

$$
R_{n}=f(\lambda)-\sum_{m=0}^{n} \frac{1}{m !} f^{(m)}(\mu)(\lambda-\mu)^{m}=\int_{\mu}^{\lambda} \frac{(\lambda-\nu)^{n}}{n !} f^{(n+1)}(\nu) d \nu .
$$

Take any $\omega^{\prime} \in(\omega, \mu)$ such that $\lambda \in\left(\omega^{\prime}, 2 \mu-\omega^{\prime}\right)$ and let

$$
M=\sup _{t \in \overline{\mathbb{R}}^{+}} e^{-\omega^{\prime} t} \varkappa(t) .
$$

Then $M<\infty$, by (1.1). Furthermore, by (4.1),

$$
\begin{aligned}
\left\|R_{n}\right\|_{E} & \leq\|f\|_{W_{\varkappa}(E)}\left|\int_{\mu}^{\lambda} \frac{(\lambda-\nu)^{n}}{n !}\left\|\left(\frac{\partial}{\partial \nu}\right)^{n+1} \phi_{\nu}\right\|_{1, \varkappa} d \nu\right| \\
& \leq M\|f\|_{W_{\varkappa}(E)}\left|\int_{\mu}^{\lambda} \frac{(\lambda-\nu)^{n}}{n !}\right| \int_{0}^{\infty}\left(\frac{\partial}{\partial \nu}\right)^{n+1} e^{\left(\omega^{\prime}-\nu\right) t} d t|d \nu| \\
& =M\|f\|_{W_{\varkappa}(E)}(n+1)\left|\int_{\mu}^{\lambda} \frac{(\lambda-\nu)^{n}}{\left(\nu-\omega^{\prime}\right)^{n+2}} d \nu\right|
\end{aligned}
$$

and hence it remains to check that

$$
\lim _{n \rightarrow \infty}(n+1) \int_{\mu}^{\lambda} \frac{(\lambda-\nu)^{n}}{\left(\nu-\omega^{\prime}\right)^{n+2}} d \nu=0
$$

Put

Then

$$
\tau=\frac{\lambda-\nu}{\nu-\omega^{\prime}}, \quad \theta=\frac{\lambda-\mu}{\mu-\omega^{\prime}} .
$$

$$
\frac{1}{\nu-\omega^{\prime}}=\frac{\tau+1}{\lambda-\omega^{\prime}}, \quad|\theta|<1
$$

and

$$
\begin{aligned}
(n+1)\left|\int_{\mu}^{\lambda} \frac{(\lambda-\nu)^{n}}{\left(\nu-\omega^{\prime}\right)^{n+2}} d \nu\right| & =(n+1)\left|\int_{\mu}^{\lambda}\left(\frac{\lambda-\nu}{\nu-\omega^{\prime}}\right)^{n} d \frac{1}{\nu-\omega^{\prime}}\right| \\
& =\frac{n+1}{\lambda-\omega^{\prime}}\left|\int_{0}^{\theta} \tau^{n} d \tau\right|=\frac{|\theta|^{n+1}}{\lambda-\omega^{\prime}}
\end{aligned}
$$

whence (4.2) follows $\left({ }^{4}\right)$.

$\left({ }^{4}\right)$ Lemma 4.1 and its proof, due to the present author, were first published in [B] in the case of $\varkappa(t)=e^{\omega t}$. 
The following lemma refers to the calculations on p. 21 of $[\mathrm{G}]$.

LEMMA 4.2. Let $f$ be a real analytic map from an interval $I \subset(\omega, \infty)$ to a Banach space $E$. For every $\lambda \in I$ put

$$
|f|_{\lambda}=\sup \left\{\frac{\left\|(\partial / \partial \lambda)^{n} f(\lambda)\right\|_{E}}{\left\|(\partial / \partial \lambda)^{n} \phi_{\lambda}\right\|_{1, \varkappa}}: n=0,1, \ldots\right\} .
$$

Then the $[0, \infty]$-valued function $\lambda \rightarrow|f|_{\lambda}$ increases on $I$.

Proof. Lemma 4.2 will follow once it is shown that if $|f|_{\mu}<\infty$ for some $\mu \in I$, then $|f|_{\lambda} \leq|f|_{\mu}$ for every $\lambda \in I \cap(\omega, \mu]$. So, suppose that $\mu \in I$ and $|f|_{\mu}<\infty$. Since $\phi_{\bullet} \in W_{\varkappa}\left(L_{\varkappa}^{1}\left(\mathbb{R}^{+}\right)\right)$, it follows from Lemma 4.1 that

$$
\left(\frac{\partial}{\partial \lambda}\right)^{n} \phi_{\lambda}=\sum_{m=0}^{\infty} \frac{(\lambda-\mu)^{m}}{m !}\left(\frac{\partial}{\partial \mu}\right)^{n+m} \phi_{\mu}
$$

for every $n=0,1, \ldots$ and $\lambda \in(\omega, 2 \mu-\omega)$, the series being absolutely convergent in the norm of $L_{\varkappa}^{1}\left(\mathbb{R}^{+}\right)$. Hence, by real analyticity of $f$,

$$
\begin{aligned}
\left\|\left(\frac{\partial}{\partial \lambda}\right)^{n} f(\lambda)\right\|_{E} & \leq \sum_{m=0}^{\infty}\left\|\frac{(\lambda-\mu)^{m}}{m !}\left(\frac{\partial}{\partial \mu}\right)^{n+m} f(\mu)\right\|_{E} \\
& \leq|f|_{\mu} \sum_{m=0}^{\infty}\left\|\frac{(\lambda-\mu)^{m}}{m !}\left(\frac{\partial}{\partial \mu}\right)^{n+m} \phi_{\mu}\right\|_{1, \varkappa}<\infty
\end{aligned}
$$

for all $n=0,1, \ldots$ and $\lambda \in I \cap(\omega, 2 \mu-\omega)$. If $\lambda \in I \cap(\omega, \mu]$ then

$$
(-1)^{n} \frac{(\lambda-\mu)^{m}}{m !}\left(\frac{\partial}{\partial \mu}\right)^{n+m} \phi_{\mu}(t)=\frac{t^{n+m}(\mu-\lambda)^{m}}{m !} e^{-\mu t} \geq 0
$$

for all $t \in \mathbb{R}^{+}$and $m=0,1, \ldots$, so that

$$
\begin{aligned}
\sum_{m=0}^{\infty}\left\|\frac{(\lambda-\mu)^{m}}{m !}\left(\frac{\partial}{\partial \mu}\right)^{n+m} \phi_{\mu}\right\|_{1, \varkappa} & =\left\|\sum_{m=0}^{\infty} \frac{(\lambda-\mu)^{m}}{m !}\left(\frac{\partial}{\partial \mu}\right)^{n+m} \phi_{\mu}\right\|_{1, \varkappa} \\
& =\left\|\left(\frac{\partial}{\partial \lambda}\right)^{n} \phi_{\lambda}\right\|_{1, \varkappa} \cdot
\end{aligned}
$$

Therefore, if $\lambda \in I \cap(\omega, \mu]$, then $\left\|(\partial / \partial \lambda)^{n} f(\lambda)\right\|_{E} \leq|f|_{\mu}\left\|(\partial / \partial \lambda)^{n} \phi_{\lambda}\right\|_{1, \varkappa}$ for every $n=0,1, \ldots$, which means that $|f|_{\lambda} \leq|f|_{\mu}$.

LEMma 4.3. If $E$ is a Banach space, $\omega^{\prime} \in(\omega, \infty)$, and $f_{0}:\left(\omega^{\prime}, \infty\right) \rightarrow E$ is an infinitely differentiable map such that $\sup _{\lambda \in\left(\omega^{\prime}, \infty\right)}\left|f_{0}\right|_{\lambda}<\infty$, then there is a unique map $f \in W_{\varkappa}(E)$ such that $\left.f\right|_{\left(\omega^{\prime}, \infty\right)}=f_{0}$.

Pr o of. The same argument as in the proof of Lemma 4.1 shows that the map $f_{0}$ is real analytic on $\left(\omega^{\prime}, \infty\right)$. Take any $\mu \in\left(\omega^{\prime}, \infty\right)$. Since $\left|f_{0}\right|_{\mu}<\infty$ 
and $\phi_{\bullet} \in W_{\varkappa}\left(L_{\varkappa}^{1}\left(\mathbb{R}^{+}\right)\right)$, Lemma 4.1 implies that

$$
\sum_{m=0}^{\infty}\left\|\frac{(\lambda-\mu)^{m}}{m !}\left(\frac{\partial}{\partial \mu}\right)^{m} f_{0}(\mu)\right\|_{E} \leq\left|f_{0}\right|_{\mu} \sum_{m=0}^{\infty}\left\|\frac{(\lambda-\mu)^{m}}{m !}\left(\frac{\partial}{\partial \mu}\right)^{m} \phi_{\mu}\right\|_{1, \varkappa}<\infty
$$

for every $\lambda \in(\omega, 2 \mu-\omega)$. Hence the formula

$$
f(\lambda)= \begin{cases}\sum_{m=0}^{\infty} \frac{(\lambda-\mu)^{m}}{m !}\left(\frac{\partial}{\partial \mu}\right)^{m} f_{0}(\mu) & \text { if } \lambda \in\left(\omega, \omega^{\prime}\right] \\ f_{0}(\lambda) & \text { if } \lambda \in\left(\omega^{\prime}, \infty\right),\end{cases}
$$

determines a real analytic map $f:(\omega, \infty) \rightarrow E$ extending $f_{0}$. By Lemma 4.2, $\|f\|_{W_{\varkappa}(E)}=\sup _{\lambda \in(\omega, \infty)}|f|_{\lambda}=\sup _{\lambda \in\left(\omega^{\prime}, \infty\right)}\left|f_{0}\right|_{\lambda}<\infty$, so that $f \in W_{\varkappa}(E)$.

Let $C$ be a closed convex cone in a Banach space $E$, and let $I \subset \mathbb{R}$ be an interval. A map $f: I \rightarrow E$ will be called $C$-completely monotonic on $I$ if it is infinitely differentiable and $(-1)^{n}(\partial / \partial \lambda)^{n} f(\lambda) \in C$ for every $\lambda \in I$. This terminology follows [W;I], p. 145, and [W;II], p. 154, where $E=\mathbb{R}$ and $C=\overline{\mathbb{R}^{+}}$.

Lemma 4.4. If $f \in W_{\varkappa}(E), \omega^{\prime} \in(\omega, \infty)$, and $\left.f\right|_{\left(\omega^{\prime}, \infty\right)}$ is $C$-completely monotonic on $\left(\omega^{\prime}, \infty\right)$, then $f$ is $C$-completely monotonic on $(\omega, \infty)$.

P r o of. It is sufficient to show that if $\mu \in(\omega, \infty)$ and $(-1)^{m}(\partial / \partial \mu)^{m} f(\mu)$ $\in C$ for every $m=0,1, \ldots$, then $(-1)^{n}(\partial / \partial \lambda)^{n} f(\lambda) \in C$ for all $\lambda \in(\omega, \mu]$ and $n=0,1, \ldots$ Since $f \in W_{\varkappa}(E)$, it follows from Lemma 4.1 that

$$
\left(\frac{\partial}{\partial \lambda}\right)^{n} f(\lambda)=\sum_{m=0}^{\infty} \frac{(\lambda-\mu)^{m}}{m !}\left(\frac{\partial}{\partial \mu}\right)^{n+m} f(\mu) \quad \text { for every } \lambda \in(\omega, 2 \mu-\omega),
$$

the series being absolutely convergent in the norm of $E$. Hence if $\lambda \in(\omega, \mu]$, then

$$
(-1)^{n}\left(\frac{\partial}{\partial \lambda}\right)^{n} f(\lambda)=\sum_{m=0}^{\infty} \frac{(\mu-\lambda)^{m}}{m !}(-1)^{n+m}\left(\frac{\partial}{\partial \mu}\right)^{n+m} f(\mu) \in C .
$$

5. The main result. Let $\phi_{\bullet} \in W_{\varkappa}\left(L_{\varkappa}^{1}\left(\mathbb{R}^{+}\right)\right)$be the map defined by $(2.1)$ and let $E$ be a Banach space. For every operator $T \in L\left(L_{\varkappa}^{1}\left(\mathbb{R}^{+}\right) ; E\right)$ consider the $E$-valued function $T \phi_{\bullet}: \lambda \rightarrow T \phi_{\lambda}$ defined on $(\omega, \infty)$. The following theorem extends to continuous submultiplicative weight functions $\varkappa$ satisfying condition (1.1) the result proved earlier for $\varkappa(t)=e^{\omega t}$ by B. Hennig and F. Neubrander in $[\mathrm{H}-\mathrm{N}]$, Lemma 2.3 and Theorem 2.5.

TheOREM 5.1. The map $T \rightarrow T \phi_{\bullet}$ is an isometric isomorphism of the space of operators $L\left(L_{\varkappa}^{1}\left(\mathbb{R}^{+}\right) ; E\right)$ onto the Widder space $W_{\varkappa}(E)$.

Proof. Clearly, $T \rightarrow T \phi$. is a linear operator from $L\left(L_{\varkappa}^{1}\left(\mathbb{R}^{+}\right) ; E\right)$ into $W_{\varkappa}(E)$ with norm no greater than 1 . Therefore Theorem 1 will follow once 
it is shown that for every $f \in W_{\varkappa}(E)$ there is a unique $T \in L\left(L_{\varkappa}^{1}\left(\mathbb{R}^{+}\right) ; E\right)$ such that

$$
\|T\|_{L\left(L_{\varkappa}^{1}\left(\mathbb{R}^{+}\right) ; E\right)} \leq\|f\|_{W_{\varkappa}(E)}
$$

and

$$
T \phi_{\lambda}=f(\lambda) \quad \text { for every } \lambda \in(\omega, \infty) .
$$

The uniqueness of $T$ satisfying (5.2) follows at once from Lemma 2.1.

The existence will follow once for every $f \in W_{\varkappa}(E)$ and every $\mu \in \mathbb{R}^{+}$ we construct $T_{\mu} \in L\left(L_{\varkappa}^{1}\left(\mathbb{R}^{+}\right) ; E\right)$ such that

$$
\left\|T_{\mu}\right\|_{L\left(L_{\varkappa}^{1}\left(\mathbb{R}^{+}\right) ; E\right)} \leq K_{\mu}\|f\|_{W_{\varkappa}(E)}
$$

and

$$
T_{\mu} \phi_{\lambda}=\theta_{\mu} f\left(\omega+\theta_{\mu}(\lambda-\omega)\right) \quad \text { for every } \lambda \in(\omega, \infty) \text {, }
$$

where

$$
K_{\mu}=\left.\sup _{t \in \mathbb{R}^{+}}\left(\varkappa_{0}(t)\right)^{-1} \frac{\mu^{n+1}}{n !}\left\|\left(\frac{\partial}{\partial \mu}\right)^{n} \phi_{\mu+\omega}\right\|_{1, \varkappa}\right|_{n=[\mu t]}
$$

and

$$
\theta_{\mu}=\frac{\mu}{\lambda-\omega}\left(1-e^{(\omega-\lambda) / \mu}\right) .
$$

Indeed, $\lim _{\mu \rightarrow \infty} \theta_{\mu}=1$, so that by $(5.2)_{\mu}$,

$$
\lim _{\mu \rightarrow \infty} T_{\mu} \phi_{\lambda}=f(\lambda) \quad \text { for every } \lambda \in(\omega, \infty),
$$

in the norm of $E$. Furthermore, $\lim _{\mu \rightarrow \infty} K_{\mu}=1$, by (2.2), and hence (5.1) implies

$$
\limsup _{\mu \rightarrow \infty}\left\|T_{\mu}\right\|_{L\left(L_{\varkappa}^{1}\left(\mathbb{R}^{+}\right) ; E\right)} \leq\|f\|_{W_{\varkappa}(E)} .
$$

Since, by Lemma 2.1, the set $\left\{\phi_{\lambda}: \lambda \in(\omega, \infty)\right\}$ is linearly dense in $L_{\varkappa}^{1}\left(\mathbb{R}^{+}\right)$, from (5.3) and (5.4) it follows that $\lim _{\mu \rightarrow \infty} T_{\mu}=T$ exists in the strong operator topology of $L\left(L_{\varkappa}^{1}\left(\mathbb{R}^{+}\right) ; E\right)$. Also from (5.3) and (5.4) it follows that $T$ satisfies (5.1) and (5.2).

To construct $T_{\mu}$ satisfying $(5.1)_{\mu}$ and $(5.2)_{\mu}$, fix $f \in W_{\varkappa}(E)$ and $\mu \in \mathbb{R}^{+}$. For every $t \in \mathbb{R}^{+}$put

$$
g_{\mu}(t)=\left.\mu e^{\omega t} \frac{(-\mu)^{n}}{n !} f^{(n)}(\omega+\mu)\right|_{n=[\mu t]} .
$$

Then, according to (4.1),

$$
\begin{aligned}
\left\|g_{\mu}(t)\right\|_{E} & \leq\left.\|f\|_{W_{\varkappa}(E)} e^{\omega t} \frac{\mu^{n+1}}{n !}\left\|\left(\frac{\partial}{\partial \mu}\right)^{n} \phi_{\omega+\mu}\right\|_{1, \varkappa}\right|_{n=[\mu t]} \\
& =\|f\|_{W_{\varkappa}(E)} e^{\omega t} K_{\mu} \varkappa_{0}(t)=K_{\mu}\|f\|_{W_{\varkappa}(E) \varkappa(t)}
\end{aligned}
$$


for every $t \in \overline{\mathbb{R}^{+}}$. Furthermore, the $E$-valued function $t \rightarrow g_{\mu}(t)$ is piecewise continuous on $\mathbb{R}^{+}$. Therefore, by (5.6), the formula

$$
T_{\mu} \varphi=\int_{0}^{\infty} \varphi(t) g_{\mu}(t) d t, \quad \varphi \in L_{\varkappa}^{1}\left(\mathbb{R}^{+}\right),
$$

in which the integral is understood in the sense of Bochner, determines an operator $T_{\mu} \in L\left(L_{\varkappa}^{1}\left(\mathbb{R}^{+}\right) ; E\right)$ satisfying $(5.1)_{\mu}$. To check (5.2) $)_{\mu}$, take any $\lambda \in(\omega, \infty)$. Since, for every $n=0,1, \ldots$,

$$
\begin{aligned}
\mu(-\mu)^{n} \int_{n / \mu}^{(n+1) / \mu} e^{(\omega-\lambda) t} d t & =\frac{\mu(-\mu)^{n}}{\lambda-\omega}\left(1-e^{(\omega-\lambda) / \mu}\right) e^{(\omega-\lambda) n / \mu} \\
& =\theta_{\mu}\left(-\mu e^{(\omega-\lambda) / \mu}\right)^{n}
\end{aligned}
$$

and since $\left|-\mu e^{(\omega-\lambda) / \mu}\right|<\mu$, from (5.5), (5.7) and Lemma 4.1 it follows that

$$
\begin{aligned}
T_{\mu} \phi_{\lambda} & =\mu \sum_{n=0}^{\infty}\left(\int_{n / \mu}^{(n+1) / \mu} e^{(\omega-\lambda) t} d t\right) \frac{(-\mu)^{n}}{n !} f^{(n)}(\omega+\mu) \\
& =\theta_{\mu} \sum_{n=0}^{\infty} \frac{\left(-\mu e^{(\omega-\lambda) / \mu}\right)^{n}}{n !} f^{(n)}(\omega+\mu) \\
& =\theta_{\mu} f\left(\omega+\mu-\mu e^{(\omega-\lambda) / \mu}\right)=\theta_{\mu} f\left(\omega+\theta_{\mu}(\lambda-\omega)\right) .
\end{aligned}
$$

Thus $(5.2)_{\mu}$ is satisfied.

The main idea of the above proof is similar to one of the proof of Theorem 2.5 in $[\mathrm{H}-\mathrm{N}]$. The difference lies in another choice of the approximating operators: the operators (5.7) defined by means of the functions (5.5) replace the operators $\widetilde{T}_{k}: L^{1}\left(\mathbb{R}^{+}\right) \ni \varphi \rightarrow \int_{0}^{\infty} \varphi(t) \widetilde{g}_{k}(t) d t \in E$ defined on p. 160 of $[\mathrm{H}-\mathrm{N}]$ by means of the functions

$$
\widetilde{g}_{k}(t)=\frac{(-1)^{k}}{k !}\left(\frac{k}{t}\right)^{k+1} f^{(k)}\left(\frac{k}{t}\right), \quad t \in \mathbb{R}^{+}, k=1,2, \ldots
$$

In the scalar case the functions (5.8) occur in the Post-Widder inversion formula for the Laplace transform, and in the "general representation theorem" of Widder (Theorem 11a of Chapter VII of [W;I], p. 303). If $\omega=0$, $\varkappa \equiv 1, X$ is a Banach space, $E=L(X)$, and $\mathbb{R}^{+} \ni \lambda \rightarrow f(\lambda)=R_{\lambda} \in L(X)$ is the resolvent of a bounded $C_{0}$ semigroup $\left(S_{t}\right)_{t \in \overline{\mathbb{R}^{+}}} \subset L(X)$, then

(i) $\widetilde{g}_{k}(t)=\left(\frac{k}{t} R_{k / t}\right)^{k+1}$ for $t \in \mathbb{R}^{+}$and $k=1,2, \ldots$, which is related to the E. Hille approximation formula 


$$
\lim _{k \rightarrow \infty} \sup _{t \in[0, b]}\left\|\left(\frac{k}{t} R_{k / t}\right)^{k} x-S_{t} x\right\|_{X}=0
$$

for all $x \in X$ and $b \in \mathbb{R}^{+}$,

(ii) $g_{\mu}(t)=\left(\mu R_{\mu}\right)^{[\mu t]+1}$ for $t \in \overline{\mathbb{R}^{+}}$and $\mu \in \mathbb{R}^{+}$, so that

$$
\lim _{\mu \rightarrow \infty} \sup _{t \in[0, b]}\left\|\left(\mu R_{\mu}\right)^{[\mu t]+1} x-S_{t} x\right\|_{X}=0
$$

for all $x \in X$ and $b \in \mathbb{R}^{+}$(by Theorem 3.6 of $[\mathrm{K} ; \mathrm{II}]$, Sec. IX.3.3, or Theorem 6.5 of $[\mathrm{E}-\mathrm{K}]$, p. 31, or Theorem 6.7 of $[\mathrm{P}]$, p. 96).

The formula (5.9) is related to E. Hille's proof of the Hille-Yosida generation theorem (see $[\mathrm{H}]$, p. 238; $[\mathrm{H}-\mathrm{P}]$, p. 362; [K;II], Sec. IX.1.2; $[\mathrm{P}]$, pp. 33-35). The formula (5.10) is related to the "implicit scheme" of the finite difference approximation to the solutions of the ACP $([\mathrm{Kr}]$, pp. 350-351), and was used by H. Trotter in his proof of the Trotter-Kato approximation theorem for $C_{0}$ semigroups ([T]; [Y;II], pp. 270-271). In [B] a formula analogous to (5.5) and (5.8) has the form

$$
\widetilde{\widetilde{g}}_{\mu}(t)=-e^{-\mu t} \sum_{n=0}^{\infty} \frac{\left(-\mu^{2} t\right)^{n+1}}{n !(n+1) !} f^{(n)}(\mu) .
$$

The expression on the right side of (5.11) occurs in R. S. Phillips' $[\mathrm{P}]$ inversion formula for the Laplace transform. See $[\mathrm{H}-\mathrm{P}]$, p. 221, Theorem 6.6.3. If $X$ is a Banach space and $\mathbb{R}^{+} \ni \lambda \rightarrow f(\lambda)=R_{\lambda} \in L(X)$ is the resolvent of a bounded $C_{0}$ semigroup $\left(S_{t}\right)_{t \in \overline{\mathbb{R}^{+}}} \subset L(X)$, then

(iii) $\widetilde{\widetilde{g}}_{\mu}(t)+e^{-\mu t}=e^{-\mu t} \sum_{n=0}^{\infty} \frac{t^{n}}{n !}\left(\mu^{2} R_{\mu}\right)^{n}=\exp t\left(\mu^{2} R_{\mu}-\mu\right)$ and

$$
\lim _{\mu \rightarrow \infty} \sup _{t \in[0, b]}\left\|\left[\exp t\left(\mu^{2} R_{\mu}-\mu\right)\right] x-S_{t} x\right\|_{X}=0
$$

for all $b \in \mathbb{R}^{+}$and $x \in X$, according to the approximation formula of K. Yosida ([Y;II], Remark on p. 248; [E-K], p. 14, Proposition 2.7; $[\mathrm{P}]$, p. 21, Theorem 5.5).

K. Yosida's famous proof $[\mathrm{Y} ; \mathrm{I}]$ of the Hille-Yosida generation theorem is related to the approximation (5.12). See [Y;II], pp. 246-249; [H-P], pp. 361-362; [D], pp. 48-51; [D-M;C], p. 312; [D-S;I], Sec. VIII.1, Theorem 13; [G], p. 18; [S-K], pp. 304-307; [P], pp. 8-11.

6. Complete monotonicity and positivity. Let $E$ be a Banach space and let $C \subset E$ be a closed convex cone. As in Lemma 4.4, an $E$-valued infinitely differentiable function $f$ defined on $(\omega, \infty)$ is called $C$-completely monotonic if $(-1)^{n} f^{(n)}(\lambda) \in C$ for every $n=0,1, \ldots$ and $\lambda \in(\omega, \infty)$. An operator $T \in L\left(L_{\varkappa}^{1}\left(\mathbb{R}^{+}\right) ; E\right.$ ) will be called $C$-positive if $T \varphi \in C$ for every 
real positive $\varphi \in L_{\varkappa}^{1}\left(\mathbb{R}^{+}\right)$. Let $T \rightarrow T \phi \bullet$ be the isometric isomorphism of $L\left(L_{\varkappa}^{1}\left(\mathbb{R}^{+}\right) ; E\right)$ onto $W_{\varkappa}(E)$ appearing in Theorem 5.1.

TheOrem 6.1. Let $E$ be a Banach space and let $C \subset E$ be a closed convex cone. Then the isomorphism $T \rightarrow T \phi$. maps the cone of $C$-positive operators in $L\left(L_{\varkappa}^{1}\left(\mathbb{R}^{+}\right) ; E\right)$ onto the cone of $C$-completely monotonic elements of $W_{\varkappa}(E)$.

Proof. Since $\phi_{\bullet}$ is an $\left(L_{\varkappa}^{1}\left(\mathbb{R}^{+}\right)\right)^{+}$-completely monotonic element of $W_{\varkappa}\left(L_{\varkappa}^{1}\left(\mathbb{R}^{+}\right)\right)$, it follows that if $T \in L\left(L_{\varkappa}^{1}\left(\mathbb{R}^{+}\right) ; E\right)$ is $C$-positive, then $T \phi_{\bullet} \in$ $W_{\varkappa}(E)$ is $C$-completely monotonic. It remains to show that if $f \in W_{\varkappa}(E)$ is $C$-completely monotonic then there is a $C$-positive $T \in L\left(L_{\varkappa}^{1}\left(\mathbb{R}^{+}\right) ; E\right)$ such that $f=T \phi_{\bullet}$. To this end, let us refer to the proof of Theorem 5.1. Consider the functions $g_{\mu}: \mathbb{R}^{+} \rightarrow E$ defined by (5.5) and the operators $T_{\mu}$ defined by (5.7). If $f \in W_{\varkappa}(E)$ is $C$-completely monotonic, then $g_{\mu}(t) \in C$ for every $\mu \in \mathbb{R}^{+}$, so that the operator $T_{\mu}$ is $C$-positive for every $\mu \in \mathbb{R}^{+}$. Since the cone $C$ closed, it follows that the strong limit $T=\lim _{\mu \rightarrow \infty} T_{\mu}$ is also $C$-positive.

7. Corollaries to Theorem 5.1. Let "a.e." mean "almost every" or "almost everywhere" in the sense of the one-dimensional Lebesgue measure.

Corollary 7.1 (D. V. Widder). A function $f:(\omega, \infty) \rightarrow K$ belongs to $W_{\varkappa}(K)$ if and only if there is a function $h \in L^{\infty}\left(\mathbb{R}^{+}\right)$such that

$$
\int_{0}^{\infty} e^{-\lambda t} h(t) \varkappa(t) d t=f(\lambda) \quad \text { for every } \lambda \in(\omega, \infty) .
$$

For every $f \in W_{\varkappa}(K)$ such a function $h$ is unique up to equality a.e. on $\mathbb{R}^{+}$, and

$$
\underset{t \in \mathbb{R}^{+}}{\operatorname{ess} \sup }|h(t)|=\|f\|_{W_{\varkappa}(K)} .
$$

Proof. By Theorem 5.1, $f \in W_{\varkappa}(K)$ if and only if there is a unique continuous linear functional $T$ on $L_{\varkappa}^{1}\left(\mathbb{R}^{+}\right)$such that $\|T\|=\|f\|_{W_{\varkappa}(K)}$ and $T \phi_{\lambda}=f(\lambda)$ for every $\lambda \in(\omega, \infty)$. By the F. Riesz representation theorem ([D-S;I], Sec. IV.8, Theorem 5; [S-K], p. 156, Corollary 6.1.1; [Y;II], p. 115, Example 3) for every continuous linear functional $T$ on $L_{\varkappa}^{1}\left(\mathbb{R}^{+}\right)$ there is a unique element $h$ of $L^{\infty}\left(\mathbb{R}^{+}\right)$such that $\operatorname{ess} \sup _{t \in \mathbb{R}^{+}}|h(t)|=\|T\|$ and $\int_{0}^{\infty} \varphi(t) h(t) \varkappa(t) d t=T \varphi$ for every $\varphi \in L_{\varkappa}^{1}\left(\mathbb{R}^{+}\right)$. By Lemma 2.1, this yields the assertion.

If $\omega=0$ and $\varkappa \equiv 1$ then Corollary 7.1 coincides with Widder's characterization of the Laplace transform of an element of $L^{\infty}\left(\mathbb{R}^{+}\right)$, given in Theorems 16a and $16 \mathrm{~b}$ of $[\mathrm{W} ; \mathrm{I}]$, pp. 315-316, and Theorem 8 of [W;II], p. 157. Theorem 5.1 may be viewed as a generalization of this last result of 
Widder to functions with values in a Banach space $E$. For this reason we call $W_{\varkappa}(E)$ the Widder space. Theorem 5.1, Proposition 3.1 and equality (3.8) yield the following

Corollary 7.2 (W. Arendt; B. Hennig and F. Neubrander). Let $f$ be a function defined on $(\omega, \infty)$ with values in a Banach space $E$. Then the following four conditions are equivalent:

(a) $f \in W_{\varkappa}(E)$,

(b) there is a unique operator $T \in L\left(L_{\varkappa}^{1}\left(\mathbb{R}^{+}\right) ; E\right)$ such that $f=T \phi \bullet$,

(c) there is a unique measure $G \in M_{\varkappa}(E)$ such that

$$
f(\lambda)=\int_{0}^{\infty} e^{-\lambda t} G(d t) \quad \text { for every } \lambda \in(\omega, \infty),
$$

(d) there is a unique function $g \in \operatorname{Lip}_{\varkappa}\left(\overline{\mathbb{R}^{+}} ; E\right)$ such that

$$
f(\lambda)=\lambda \int_{0}^{\infty} e^{-\lambda t} g(t) d t \quad \text { for every } \lambda \in(\omega, \infty) .
$$

If the above equivalent conditions are satisfied, then

$$
\begin{aligned}
G(B) & =T 1_{B} \quad \text { for every } B \in \mathfrak{R}, \\
g(t) & =T 1_{(0, t]} \quad \text { for every } t \in \overline{\mathbb{R}^{+}}
\end{aligned}
$$

and

$$
\|f\|_{W_{\varkappa}(E)}=\|T\|_{L\left(L_{\varkappa}^{1}\left(\mathbb{R}^{+}\right) ; E\right)}=\|G\|_{M_{\varkappa}(E)}=\|g\|_{\operatorname{Lip}_{\varkappa}\left(\overline{\mathbb{R}^{+}} ; E\right)} .
$$

The equivalence $(\mathrm{a}) \Leftrightarrow(\mathrm{d})$ of Corollary 7.2 was discovered by W. Arendt in his well known paper [A], and appeared to be a crucial result for the theory of integrated semigroups of operators. Theorem 1.1 and Corollary 1.2 of $[\mathrm{A}]$, pp. 329-330, are concerned with $\varkappa \equiv 1$ and $\varkappa(t)=e^{\omega t}$. B. Hennig and F. Neubrander in $[\mathrm{H}-\mathrm{N}]$ have deduced the result of Arendt from theorems about operators from $L^{1}\left(\mathbb{R}^{+}\right)$into $E$. Our Proposition 3.1 and Theorem 5.1 follow $[\mathrm{H}-\mathrm{N}]$. For $\varkappa(t)=(1+t)^{k}$ the equivalence $(\mathrm{a}) \Leftrightarrow(\mathrm{d})$ was established in $[\mathrm{deL}-\mathrm{H}-\mathrm{W}-\mathrm{W}]$, p. 194, Theorem 2.6, without proving that $\|g\|_{\operatorname{Lip}_{\varkappa}}=\|f\|_{W_{\varkappa}}$. In [A], [H-N] and [deL-H-W-W] difficult classical results are exploited: in $[\mathrm{A}]$ - the Widder characterization of the Laplace transform of an element of $L^{\infty}\left(\mathbb{R}^{+}\right)$; in $[\mathrm{H}-\mathrm{N}]$ - the "general representation theorem" of Widder, adapted in Lemma 2.2, p. 157, to functions with values in a Banach space; in [deL-H-W-W] - the Bernstein theorem about completely monotonic functions, used in the proof of Lemma 2.2, p. 191.

If $E$ is a Banach space then $L^{\infty}\left(\mathbb{R}^{+} ; E\right)$ denotes the space of the equivalence classes of those functions $h: \mathbb{R}^{+} \rightarrow E$ which are strongly Lebesgue measurable and essentially bounded on $\mathbb{R}^{+}$. Equipped with the norm $\|h\|_{\infty}$ 
$=\operatorname{ess}_{\sup } \operatorname{s\in \mathbb {R}}^{+}\|h(t)\|_{E}, L^{\infty}\left(\mathbb{R}^{+} ; E\right)$ is a Banach space. In the following lemma all the integrals of $E$-valued functions are understood in the Bochner sense.

LEmma 7.3. Let $E$ be a Banach space. The following four properties of $E$ are equivalent:

(i) $f \in W_{\varkappa}(E)$ if and only if there is $h \in L^{\infty}\left(\mathbb{R}^{+} ; E\right)$ such that $f(\lambda)=$ $\int_{0}^{\infty} e^{-\lambda t} \varkappa(t) h(t) d t$ for every $\lambda \in(\omega, \infty)$,

(ii) for every $g \in \operatorname{Lip}_{\varkappa}\left(\overline{\mathbb{R}^{+}} ; E\right)$ there is $h \in L^{\infty}\left(\mathbb{R}^{+} ; E\right)$ such that $g(t)=$ $\int_{0}^{t} \varkappa(u) h(u) d u$ for every $t \in \overline{\mathbb{R}^{+}}$,

(iii) for every $g \in \operatorname{Lip}_{\varkappa}\left(\overline{\mathbb{R}^{+}} ; E\right)$ the Fréchet derivative $D g(t)$ exists for a.e. $t \in \overline{\mathbb{R}^{+}}$,

(iv) for every uniformly lipschitzian function $g:[0,1] \rightarrow E$ the Fréchet derivative $D g(t)$ exists for a.e. $t \in[0,1]$,

$(\mathrm{v})$ for every absolutely continuous function $g:[0,1] \rightarrow E$ the Fréchet derivative $D g(t)$ exists for a.e. $t \in[0,1]$.

Proof. By integration by parts, the equality in (i) is equivalent to $f(\lambda)=\lambda \int_{0}^{\infty} e^{-\lambda t}\left[\int_{0}^{t} \varkappa(u) h(u) d u\right] d t$ for every $\lambda \in(\omega, \infty)$. Hence (i) $\Leftrightarrow($ ii $)$ follows from $(\mathrm{a}) \Leftrightarrow$ (d) of Corollary 7.2 by the invertibility of the Laplace transform. (ii) $\Rightarrow$ (iii) follows from Theorem 8 in Sec. III.12 of [D-S;I], or from Theorem 2 in $[\mathrm{Y} ; \mathrm{II}]$, p. 134, concerning differentiation of a Bochner integral.

If (iii) holds then there is an $h \in L^{\infty}\left(\mathbb{R}^{+} ; E\right)$ such that $D g(t)=\varkappa(t) h(t)$ for a.e. $t \in \overline{\mathbb{R}^{+}}$. Let $\widetilde{g}(t)=\int_{0}^{t} \varkappa(u) h(u) d t$. The functions $g$ and $\widetilde{g}$ are locally uniformly lipschitzian on $\overline{\mathbb{R}^{+}}, g(0)=\widetilde{g}(0)=0$ and $D g(t)=\varkappa(t) h(t)=D \widetilde{g}(t)$ for a.e. $t \in \mathbb{R}^{+}$, again by the theorem about differentiation of a Bochner integral. By Theorem 8.18 of [R;II], it follows that $l(g(t)-\widetilde{g}(t))=0$ for every $t \in \overline{\mathbb{R}^{+}}$and every linear functional $l \in E^{*}$. Hence $g=\widetilde{g}$ by the Hahn-Banach theorem, proving (iii) $\Rightarrow$ (ii).

(iii) $\Leftrightarrow\left(\right.$ iv) is a consequence of the fact that for any $b \in \mathbb{R}^{+}$the restriction $\left.g\right|_{[0, b]}$ of $g \in \operatorname{Lip}_{\varkappa}\left(\mathbb{R}^{+} ; E\right)$ may be an arbitrary uniformly Lipschitzian $E$-valued function on $[0, b]$. (v) $\Rightarrow$ (iv) is obvious.

Finally, (iv) $\Rightarrow(\mathrm{v})$ is a consequence of the Lebesgue theorem about differentiability a.e. of a scalar absolutely continuous function ([R;II], Theorem 8.18). Indeed, suppose that $g:[0,1] \rightarrow E$ is absolutely continuous and let $\left.v(t)=t+\sup _{\pi} \sum_{i=1}^{n(\pi)} \| g\left(t_{i}\right)\right)-g\left(t_{i-1}\right) \|_{E}$, the supremum being taken over all partitions $\pi: 0=t_{0}<t_{1}<\ldots<t_{n(\pi)}=t$ of $[0, t]$. Then $v:[0,1] \rightarrow \overline{\mathbb{R}^{+}}$is increasing and absolutely continuous, and $v^{-1}$ : $[0, v(1)] \rightarrow[0,1]$ and $\widetilde{g}=g \circ v^{-1}:[0, v(1)] \rightarrow E$ are uniformly lipschitzian. By the Lebesgue theorem, the derivative $D v(t)$ exists for every $t \in[0,1] \backslash N$, where $N$ is a null set for the Lebesgue measure. If (iv) holds, then the Fréchet derivative $D \widetilde{g}(u)$ exists for every $u \in[0, v(1)] \backslash M$, where $M$ is 
a null set. Hence the Fréchet derivative $D g(t)=D \widetilde{g}(v(t)) \cdot D v(t)$ exists for every $t \in[0,1] \backslash\left(N \cup v^{-1}(M)\right)$. Since $v^{-1}$ is uniformly lipschitzian, $v^{-1}(M)$ is a null set. Hence the Fréchet derivative $D g(t)$ exists for a.e. $t \in[0,1]$.

According to the definition given in [D-U], pp. 106-107, a Banach space $E$ is called a Gelfand space if the condition (v) from Lemma 7.3 is satisfied. A Banach space is a Gelfand space if and only if it has the Radon-Nikodym property $\left(^{5}\right)$.

Corollary 7.4 ([A], Theorem 1.4, p. 331). Corollary 7.1 remains true for functions taking values in a Banach space $E$ if and only if $E$ is a Gelfand space.

Let $X$ denote a Banach space. Applications of Corollary 7.2 in the theory of operator semigroups and integrated semigroups are concerned with $E=L(X)$, the Banach algebra of continuous linear endomorphisms of $X$. In this connection consider a bounded $C_{0}$ semigroup $(s(t))_{t \in \overline{\mathbb{R}}^{+}} \subset L(X)$. Then for every $t \in \overline{\mathbb{R}^{+}}$there is an operator $g(t) \in L(X)$ such that

$$
g(t) x=\int_{0}^{t} s(u) x d u \quad \text { for every } x \in X .
$$

The $L(X)$-valued function $t \rightarrow g(t)$ belongs to $\operatorname{Lip}_{\varkappa}\left(\overline{\mathbb{R}^{+}} ; L(X)\right)$ for $\varkappa \equiv 1$.

Lemma 7.5. If for a.e. $t \in \overline{\mathbb{R}^{+}}$the derivative $D g(t)$ exists in the norm topology of $L(X)$, then the semigroup $(s(t))_{t \in \overline{\mathbb{R}}^{+}}$is continuous on the open halfaxis $\mathbb{R}^{+}$in this topology.

Proof. The assumptions imply that

$$
\lim _{n \rightarrow \infty}\|n(g(t+1 / n)-g(t))-s(t)\|=0 \quad \text { for a.e. } t \in \overline{\mathbb{R}^{+}},
$$

whence it follows that the $L(X)$-valued function $t \rightarrow s(t)$ is strongly measurable on $\overline{\mathbb{R}^{+}}$with respect to the Lebesgue measure ([Y;II $]$, p. 130). From now on we follow an argument of N. Dunford [D]. (See [D-S;I], Sec. VIII.1, proof of Lemma 3 ; [H-P], p. 305, Th. 10.2.3; [Y;II], p. 234). Fix any $t_{0} \in \mathbb{R}^{+}$ and put $d=\frac{1}{2} t_{0}$. Then

$(*) \quad\left\|s\left(t_{0}+h\right)-s\left(t_{0}\right)\right\|=\left\|s\left(t_{0}-t\right)[s(t+h)-s(t)]\right\| \leq M\|s(t+h)-s(t)\|$ for all $t \in[d, 2 d]$ and $h \in[-d, d]$, where $M=\sup _{t \in \mathbb{R}^{+}}\|s(t)\|$. The strong Lebesgue measurability of the bounded $L(X)$-valued function $t \rightarrow s(t)$ implies that for any fixed $h \in[-d, d]$ the bounded positive function $t \rightarrow$

$\left({ }^{5}\right)$ In [D-U], the Radon-Nikodym property is defined on p. 61 , and its equivalence with the Gelfand property is not stated explicitly, but follows from Theorem 2 on p. 107, Theorem 7 on p. 136, and Corollary 8 on p. 138. 
$\|s(t+h)-s(t)\|$ is Lebesgue measurable on $[d, 2 d]$ and

$$
\lim _{h \rightarrow 0} \int_{d}^{2 d}\|s(t+h)-s(t)\| d t=0 .
$$

(See $[\mathrm{H}-\mathrm{P}]$, p. 86 , Theorem 3.83.) The estimate $(*)$ implies that

$$
\left\|s\left(t_{0}+h\right)-s\left(t_{0}\right)\right\| \leq \frac{M}{d} \int_{d}^{2 d}\|s(t+h)-s(t)\| d t \quad \text { whenever } h \in[-d, d] .
$$

Corollary 7.6. If $X$ is a Banach space such that there exists a $C_{0}$ semigroup $(s(t))_{t \in \overline{\mathbb{R}^{+}}} \subset L(X)$ which is not continuous on $\mathbb{R}^{+}$in the norm topology of $L(X)$, then $L(X)$ is not a Gelfand space.

8. Convolution and the semigroup of right translations in $L_{\varkappa}^{1}\left(\mathbb{R}^{+}\right)$. This section contains some basic material needed in the sequel. The proofs are standard and are included only for completeness.

8.1. Convolution in $L_{\varkappa}^{1}\left(\mathbb{R}^{+}\right)$. Let $\varphi$ and $\psi$ be elements of $L_{\varkappa}^{1}\left(\mathbb{R}^{+}\right)$represented by functions $\varphi^{0}$ and $\psi^{0}$. Then the function $(s, t) \rightarrow \varphi^{0}(s) \psi^{0}(t-s)$ is Lebesgue measurable on $\Delta=\left\{(s, t) \in \mathbb{R}^{2}: 0<s<t<\infty\right\}$. By submultiplicativity of $\varkappa$, and by the Fubini-Tonelli theorem,

$$
\begin{aligned}
\iint_{\Delta}\left|\varphi^{0}(s) \psi^{0}(t-s)\right| \varkappa(t) d s d t \\
\quad \leq \iint_{\Delta}\left|\varphi^{0}(s) \psi^{0}(t-s)\right| \varkappa(s) \varkappa(t-s) d s d t \\
\quad=\int_{0}^{\infty}\left|\varphi^{0}(s) \varkappa(s)\right|\left[\int_{s}^{\infty}\left|\psi^{0}(t-s) \varkappa(t-s)\right| d t\right] d s \\
\quad=\|\varphi\|_{1, \varkappa}\|\psi\|_{1, \varkappa}<\infty,
\end{aligned}
$$

so that the set

$$
N=\left\{t \in \mathbb{R}^{+}: \int_{0}^{t}\left|\varphi^{0}(s) \psi^{0}(t-s)\right| d s=\infty\right\}
$$

has one-dimensional Lebesgue measure zero. Again by the Fubini-Tonelli theorem, it follows that

$$
t \rightarrow \eta(t)=1_{\mathbb{R}^{+} \backslash N}(t) \int_{0}^{t} \varphi^{0}(s) \psi^{0}(t-s) d s=1_{\mathbb{R}^{+} \backslash N}(t) \int_{0}^{t} \varphi(s) \psi(t-s) d s
$$

is a finite-valued function Lebesgue measurable on $\mathbb{R}^{+}$such that

$$
\int_{0}^{\infty}|\eta(t)| \varkappa(t) d t=\iint_{\Delta}\left|\varphi^{0}(s) \psi^{0}(t-s)\right| \varkappa(t) d s d t \leq\|\varphi\|_{1, \varkappa}\|\psi\|_{1, \varkappa} .
$$


The element of $L_{\varkappa}^{1}\left(\mathbb{R}^{+}\right)$represented by $\eta$ depends only on $\varphi$ and $\psi$, is denoted by $\varphi * \psi$, and is called the convolution of $\varphi$ and $\psi$. Evidently,

$$
\|\varphi * \psi\|_{1, \varkappa} \leq\|\varphi\|_{1, \varkappa}\|\psi\|_{1, \varkappa}
$$

and

$$
\varphi * \psi=\psi * \varphi
$$

for all $\varphi$ and $\psi$ in $L_{\varkappa}^{1}\left(\mathbb{R}^{+}\right)$. Furthermore,

$$
(\varphi * \psi) * \zeta=\varphi *(\psi * \zeta)
$$

for all $\varphi, \psi$ and $\zeta$ in $L_{\varkappa}^{1}\left(\mathbb{R}^{+}\right)$. Indeed, if $\varphi, \psi$ and $\zeta$ are represented by functions $\varphi^{0}, \psi^{0}$ and $\zeta^{0}$ then, similarly to the case of $N$, the set

$$
M=\left\{t \in \mathbb{R}^{+}: \int_{0}^{t}\left(\left|\varphi^{0}\right| *\left|\psi^{0}\right|\right)(s)\left|\zeta^{0}(t-s)\right| d s=\infty\right\}
$$

has one-dimensional Lebesgue measure zero. Furthermore, for every $t \in$ $\mathbb{R}^{+} \backslash M$ the function $(r, s) \rightarrow \varphi^{0}(r) \psi^{0}(s-r) \zeta^{0}(t-s)$ is Lebesgue measurable on $\Delta_{t}=\left\{(r, s) \in \mathbb{R}^{2}: 0<r<s<t\right\}$, and by the Fubini-Tonelli theorem,

$$
\iint_{\Delta_{t}}\left|\varphi^{0}(r) \psi^{0}(s-r) \zeta^{0}(t-s)\right| d r d s=\int_{0}^{t}\left(\left|\varphi^{0}\right| *\left|\psi^{0}\right|\right)(s)\left|\zeta^{0}(t-s)\right| d s<\infty .
$$

Thus, again by the Fubini-Tonelli theorem, if $t \in \mathbb{R}^{+} \backslash M$, then

$$
\begin{aligned}
\int_{0}^{t}[\varphi * \psi](s) \zeta(t-s) d s & =\int_{0}^{t}\left[\int_{0}^{s} \varphi^{0}(r) \psi^{0}(s-r) d r\right] \zeta^{0}(t-s) d s \\
& =\iint_{\Delta_{t}} \varphi^{0}(r) \psi^{0}(s-r) \zeta^{0}(t-s) d r d s \\
& =\int_{0}^{t} \varphi^{0}(r)\left[\int_{r}^{t} \psi^{0}(s-r) \zeta^{0}(t-s) d s\right] d r \\
& =\int_{0}^{t} \varphi^{0}(r)\left[\int_{0}^{t-r} \psi^{0}(u) \zeta^{0}(t-r-u) d u\right] d r \\
& =\int_{0}^{t} \varphi(r)[\psi * \zeta](t-r) d r
\end{aligned}
$$

which yields (8.4).

It is obvious that the convolution operator $*: L_{\varkappa}^{1}\left(\mathbb{R}^{+}\right) \times L_{\varkappa}^{1}\left(\mathbb{R}^{+}\right) \rightarrow$ $L_{\varkappa}^{1}\left(\mathbb{R}^{+}\right)$is bilinear. Hence conditions (8.2), (8.3) and (8.4) mean that $L_{\varkappa}^{1}\left(\mathbb{R}^{+}\right)$is a convolution Banach algebra.

8.2. Right translations in $L_{\varkappa}^{1}\left(\mathbb{R}^{+}\right)$. If $\varphi^{0}$ is a function on $\mathbb{R}^{+}$then for every $t \in \overline{\mathbb{R}^{+}}$the right translate of $\varphi^{0}$ by $t$ is, by definition, the function $\varphi_{t}^{0}$ 
on $\mathbb{R}^{+}$such that

$$
\varphi_{t}^{0}(s)= \begin{cases}0 & \text { if } s \in(0, t] \\ \varphi^{0}(s-t) & \text { if } s \in(t, \infty)\end{cases}
$$

If $t \in \overline{\mathbb{R}^{+}}$and $\varphi$ is an element of $L_{\varkappa}^{1}\left(\mathbb{R}^{+}\right)$represented by a function $\varphi^{0}$, then

$$
\begin{aligned}
\int_{0}^{\infty}\left|\varphi_{t}^{0}(s)\right| \varkappa(s) d s & =\int_{t}^{\infty}\left|\varphi^{0}(s-t)\right| \varkappa(s) d s=\int_{0}^{\infty}\left|\varphi^{0}(u)\right| \varkappa(t+u) d u \\
& \leq \varkappa(t) \int_{0}^{\infty}\left|\varphi^{0}(u)\right| \varkappa(u) d u=\varkappa(t)\|\varphi\|_{1, \varkappa},
\end{aligned}
$$

so that there is a unique element $\varphi_{t}$ of $L_{\varkappa}^{1}\left(\mathbb{R}^{+}\right)$represented by $\varphi_{t}^{0}$. This element $\varphi_{t}$ depends only on $\varphi$ and $t$, and is called the right translate of $\varphi$ by $t$. The preceding estimate shows that

$$
\left\|\varphi_{t}\right\|_{1, \varkappa} \leq \varkappa(t)\|\varphi\|_{1, \varkappa}
$$

for all $\varphi \in L_{\varkappa}^{1}\left(\mathbb{R}^{+}\right)$and $t \in \overline{\mathbb{R}^{+}}$. As a consequence, for every $t \in \mathbb{R}^{+}$the operator $U_{t}$ of right translation by $t$, i.e. the operator $U_{t}: \varphi \rightarrow \varphi_{t}$, belongs to $L\left(L_{\varkappa}^{1}\left(\mathbb{R}^{+}\right)\right)$and

$$
\left\|U_{t}\right\|_{L\left(L_{\varkappa}^{1}\left(\mathbb{R}^{+}\right)\right)} \leq \varkappa(t) .
$$

The right translation operators constitute a one-parameter semigroup. Since the set $\left\{1_{(0, a]}: a \in \mathbb{R}^{+}\right\}$is linearly dense in $L_{\varkappa}^{1}\left(\mathbb{R}^{+}\right)$and

$$
\left\|U_{t} 1_{(0, a]}-1_{(0, a]}\right\|_{1, \varkappa}=\left\|1_{(a, a+t]}-1_{(0, t]}\right\|_{1, \varkappa} \leq\left(\int_{a}^{a+t}+\int_{0}^{t}\right) \varkappa(u) d u,
$$

it follows by (8.5) that

$$
\lim _{t \downarrow 0}\left\|U_{t} \varphi-\varphi\right\|_{1, \varkappa}=0
$$

for every $\varphi \in L_{\varkappa}^{1}\left(\mathbb{R}^{+}\right)$. Conditions (8.5) and (8.6) imply that for every $\varphi \in L_{\varkappa}^{1}\left(\mathbb{R}^{+}\right)$the map $\overline{\mathbb{R}^{+}} \ni t \rightarrow U_{t} \varphi \in L_{\varkappa}^{1}\left(\mathbb{R}^{+}\right)$is continuous in the norm of $L_{\varkappa}^{1}\left(\mathbb{R}^{+}\right)$(see [Y;II], p. 233; [D], p. 13; [G], p. 22, Exercise 2.18.1). Hence $\left(U_{t}\right)_{t \in \mathbb{R}^{+}} \subset L\left(L_{\varkappa}^{1}\left(\mathbb{R}^{+}\right)\right)$is a $C_{0}$ semigroup.

8.3. Convolution in $L_{\varkappa}^{1}\left(\mathbb{R}^{+}\right)$as a Bochner integral. If $\varphi$ and $\psi$ are in $L_{\varkappa}^{1}\left(\mathbb{R}^{+}\right)$, and $\varphi$ is represented by a function $\varphi^{0}$, then the $L_{\varkappa}^{1}\left(\mathbb{R}^{+}\right)$-valued function $\mathbb{R}^{+} \ni t \rightarrow \varphi^{0}(t) U_{t} \psi \in L_{\varkappa}^{1}\left(\mathbb{R}^{+}\right)$is strongly Lebesgue measurable [Y;II; p. 130], and, by (8.5),

$$
\int_{0}^{\infty}|\varphi(t)|\left\|U_{t} \psi\right\|_{1, \varkappa} d t \leq \int_{0}^{\infty}|\varphi(t)| \varkappa(t)\|\psi\|_{1, \varkappa} d t=\|\varphi\|_{1, \varkappa}\|\psi\|_{1, \varkappa} .
$$

Hence the Bochner integral $\int_{0}^{\infty} \varphi(t) U_{t} \psi d t$ exists. 
LEMma 8.3.1. If $\varphi$ and $\psi$ are in $L_{\varkappa}^{1}\left(\mathbb{R}^{+}\right)$, then $\varphi * \psi=\int_{0}^{\infty} \varphi(t) U_{t} \psi d t$, the integral (of the equivalence class of $L_{\varkappa}^{1}\left(\mathbb{R}^{+}\right)$-valued functions) being understood in the sense of Bochner.

Proof. It is sufficient to show that

$$
\int_{B}\left[\int_{0}^{\infty} \varphi(t) U_{t} \psi d t\right](s) d s=\int_{B}(\varphi * \psi)(s) d s
$$

for all $\varphi$ and $\psi$ in $L_{\varkappa}^{1}\left(\mathbb{R}^{+}\right)$and for every bounded Lebesgue measurable subset $B$ of $\mathbb{R}^{+}$. Since $\varphi \rightarrow \int_{B} \varphi(s) d s$ is a continuous linear functional on $L_{\varkappa}^{1}\left(\mathbb{R}^{+}\right)$, by Corollary 2 of $[\mathrm{Y} ; \mathrm{II}]$, p. 134 , it follows that

$$
\begin{aligned}
\int_{B}\left[\int_{0}^{\infty} \varphi(t) U_{t} \psi d t\right](s) d s & =\int_{0}^{\infty} \varphi(t)\left[\int_{B}\left[U_{t} \psi\right](s) d s\right] d t \\
& =\int_{0}^{\infty} \varphi(t)\left[\int_{B} \psi_{t}(s) d s\right] d t .
\end{aligned}
$$

Let $\varphi^{0}$ and $\psi^{0}$ be any functions representing $\varphi$ and $\psi$. Then the function $(t, s) \rightarrow \varphi^{0}(t) \psi^{0}(s-t) 1_{B}(s)$ is Lebesgue measurable on $\Delta=\{(t, s): 0<$ $t<s<\infty\}$, and by the Fubini-Tonelli theorem,

$$
\begin{aligned}
\iint_{\Delta}\left|\varphi^{0}(t) \psi^{0}(s-t)\right| 1_{B}(s) d s d t & =\int_{B}\left[\int_{0}^{s}\left|\varphi^{0}(t) \psi^{0}(s-t)\right| d t\right] d s \\
& \leq\left(\inf _{s \in B} \varkappa(s)\right)^{-1}\||\varphi| *|\psi|\|_{1, \varkappa} \\
& \leq\left(\inf _{s \in B} \varkappa(s)\right)^{-1}\|\varphi\|_{1, \varkappa}\|\psi\|_{1, \varkappa}<\infty .
\end{aligned}
$$

Hence, again by the Fubini-Tonelli theorem,

$$
\begin{aligned}
\int_{0}^{\infty} \varphi(t)\left[\int_{B} \psi_{t}(s) d s\right] d t & =\int_{0}^{\infty} \varphi^{0}(t)\left[\int_{t}^{\infty} \psi^{0}(s-t) 1_{B}(s) d s\right] d t \\
& =\iint_{\Delta} \varphi^{0}(t)\left[\psi^{0}(s-t) 1_{B}(s)\right] d s d t \\
& =\int_{0}^{\infty} 1_{B}(s)\left[\int_{0}^{s} \varphi^{0}(t) \psi^{0}(s-t) d t\right] d s \\
& =\int_{B}(\varphi * \psi)(s) d s .
\end{aligned}
$$

The equality (8.7) follows from (8.8) and (8.9). 
Corollary 8.3.2. If $\varphi \in L_{\varkappa}^{1}\left(\mathbb{R}^{+}\right)$and $t \in \overline{\mathbb{R}^{+}}$, then

$$
\int_{0}^{t} U_{s} \varphi d s=\int_{0}^{\infty} 1_{(0, t]}(s) U_{s} \varphi d s=1_{(0, t]} * \varphi
$$

where the function $\overline{\mathbb{R}^{+}} \ni s \rightarrow U_{s} \varphi \in L_{\varkappa}^{1}\left(\mathbb{R}^{+}\right)$is continuous in the norm of $L_{\varkappa}^{1}\left(\mathbb{R}^{+}\right)$. Hence

$$
U_{t} \varphi=\frac{d}{d t}\left[1_{(0, t]} * \varphi\right]
$$

for all $t \in \overline{\mathbb{R}^{+}}$and $\varphi \in L_{\varkappa}^{1}\left(\mathbb{R}^{+}\right)$, the derivative being computed in the norm of $L_{\varkappa}^{1}\left(\mathbb{R}^{+}\right)$.

8.4. A bounded approximate unit in $L_{\varkappa}^{1}\left(\mathbb{R}^{+}\right)$. As a consequence of Lemma 8.3.1,

$$
\int_{0}^{\infty} e^{-\lambda t} U_{t} \varphi d t=\phi_{\lambda} * \varphi
$$

for all $\lambda \in(\omega, \infty)$ and $\varphi \in L_{\varkappa}^{1}\left(\mathbb{R}^{+}\right)$, which means that the map $(\omega, \infty) \ni \lambda \rightarrow$ $\phi_{\lambda} * \in L\left(L_{\varkappa}^{1}\left(\mathbb{R}^{+}\right)\right)$is the resolvent of the semigroup $\left(U_{t}\right)_{t \in \overline{\mathbb{R}^{+}}} \subset L\left(L_{\varkappa}^{1}\left(\mathbb{R}^{+}\right)\right)$. Since this semigroup is of class $C_{0}$, its resolvent is regular ([D-M;C], p. 310), that is,

$$
\lim _{\lambda \rightarrow \infty}\left\|\lambda \phi_{\lambda} * \varphi-\varphi\right\|_{1, \varkappa}=0
$$

for every $\varphi \in L_{\varkappa}^{1}\left(\mathbb{R}^{+}\right)$. Furthermore, from continuity of the map (2.1), and from (2.3), it follows that

$$
\sup _{\lambda \in[\omega+\varepsilon, \infty)}\left\|\lambda \phi_{\lambda}\right\|_{1, \varkappa}<\infty
$$

for every $\varepsilon>0$. Conditions (8.12) and (8.13) mean that, for every $\varepsilon>0$, the net $\left(\lambda \phi_{\lambda}\right)_{\lambda \in[\omega+\varepsilon, \infty)}$ is a bounded approximate unit ([H-R;II], p. 87; [Pal], p. 520) in the commutative convolution Banach algebra $L_{\varkappa}^{1}\left(\mathbb{R}^{+}\right)$.

9. Homomorphisms of the convolution algebra $L_{\varkappa}^{1}\left(\mathbb{R}^{+}\right)$into a Banach algebra $A$ and pseudoresolvents in $W_{\varkappa}(A)$. Let $A$ be a Banach algebra. A pseudoresolvent defined on $(\omega, \infty)$ and taking values in $A$ is by definition a map $r:(\omega, \infty) \rightarrow A$ satisfying the resolvent equation

$$
r(\lambda)-r(\mu)=(\mu-\lambda) r(\lambda) r(\mu)
$$

for all $\lambda$ and $\mu$ in $(\omega, \infty)$. It is easy to see that the range of a pseudoresolvent consists of mutually commuting elements of $A$, and if $r(\lambda)=0$ for some $\lambda \in(\omega, \infty)$, then $r$ vanishes identically on $(\omega, \infty)$. An application of the Neumann series shows that every pseudoresolvent $r:(\omega, \infty) \rightarrow A$ is a real 
analytic $A$-valued function on $(\omega, \infty)$ such that

$$
\left(\frac{\partial}{\partial \lambda}\right)^{n} r(\lambda)=(-1)^{n} n ![r(\lambda)]^{n+1}
$$

for all $\lambda \in(\omega, \infty)$ and $n=1,2, \ldots$ (See [H-P], Sec. 5.8; [D-S;II], Sec. IX.1; [D-M;C], Sec. XII.5; [Y], Sec. VII.4.) If $r:(\omega, \infty) \rightarrow A$ is a pseudoresolvent then, by (9.1),

$$
\|r(\mu)\|=\lim _{\lambda \rightarrow \infty}\left\|\lambda r(\lambda)\left[\frac{1}{\lambda}+\frac{\lambda-\mu}{\lambda} r(\mu)\right]\right\| \leq\|r(\mu)\| \liminf _{\lambda \rightarrow \infty} \lambda\|r(\lambda)\|
$$

for every $\mu \in(\omega, \infty)$. Thus, if $r:(\omega, \infty) \rightarrow A$ is a non-vanishing pseudoresolvent, then $\liminf _{\lambda \rightarrow \infty} \lambda\|r(\lambda)\| \geq 1$. From this, by (2.3) and (4.1), it follows that if $r \in W_{\varkappa}(A)$ and $r$ is a non-vanishing pseudoresolvent, then $\|r\|_{W_{\varkappa}(A)} \geq 1$.

A direct computation (or an application of (8.11)) shows that

the map (2.1), i.e. the map $\phi_{\bullet}:(\omega, \infty) \ni \lambda \rightarrow \phi_{\lambda} \in L_{\varkappa}^{1}\left(\mathbb{R}^{+}\right)$is a pseudoresolvent with values in the convolution Banach algebra $L_{\varkappa}^{1}\left(\mathbb{R}^{+}\right)$.

From (9.2), (4.1) and Lemma 4.2 it follows that if $r \in W_{\varkappa}(A)$ and $r$ is a pseudoresolvent, then

$$
\begin{aligned}
\|r\|_{W_{\varkappa}(A)} & =\sup \left\{\frac{\left\|[r(\lambda)]^{n}\right\|_{A}}{\left\|\phi_{\lambda}^{* n}\right\|_{1, \varkappa}}: \lambda \in(\omega, \infty), n=1,2, \ldots\right\} \\
& =\lim _{\lambda \rightarrow \infty} \sup _{n=1,2, \ldots} \frac{\left\|[r(\lambda)]^{n}\right\|_{A}}{\left\|\phi_{\lambda}^{* n}\right\|_{1, \varkappa}} .
\end{aligned}
$$

Let $\operatorname{Hom}\left(L_{\varkappa}^{1}\left(\mathbb{R}^{+}\right) ; A\right)$ denote the set of all continuous homomorphisms of the convolution Banach algebra $L_{\varkappa}^{1}\left(\mathbb{R}^{+}\right)$into a Banach algebra $A$. If $T \in \operatorname{Hom}\left(L_{\varkappa}^{1}\left(\mathbb{R}^{+}\right) ; A\right)$, then $\|T \varphi\|=\lim _{\lambda \rightarrow \infty}\left\|T \lambda \phi_{\lambda} \cdot T \varphi\right\| \leq\|T\| \cdot\|T \varphi\|$ for every $\varphi \in L_{\varkappa}^{1}\left(\mathbb{R}^{+}\right)$, by (8.12) and (2.3). Hence if $0 \neq T \in \operatorname{Hom}\left(L_{\varkappa}^{1}\left(\mathbb{R}^{+}\right) ; A\right)$, then $\|T\|_{L\left(L_{\varkappa}^{1}\left(\mathbb{R}^{+}\right) ; A\right)} \geq 1$. From (9.3) it follows that

(9.5) if $T \in \operatorname{Hom}\left(L_{\varkappa}^{1}\left(\mathbb{R}^{+}\right) ; A\right)$, then $T \phi_{\bullet}:(\omega, \infty) \ni \lambda \rightarrow T \phi_{\bullet} \in A$ is a pseudoresolvent with values in $A$.

Theorem 9.1. Let $A$ be a Banach algebra. For every map $r:(\omega, \infty) \rightarrow$ $A$ the following two conditions are equivalent:

(a) $r$ is a pseudoresolvent and $r \in W_{\varkappa}(A)$,

(b) there is $T \in \operatorname{Hom}\left(L_{\varkappa}^{1}\left(\mathbb{R}^{+}\right) ; A\right)$ such that $r=T \phi_{\bullet}$.

If these conditions are satisfied then there is exactly one $T$ satisfying (b), and $\|T\|_{L\left(L_{\varkappa}^{1}\left(\mathbb{R}^{+}\right) ; A\right)}=\|r\|_{W_{\varkappa}(A)}$.

Proof. According to Theorem 5.1, the map $T \rightarrow T \phi \bullet$ is an isometry of $L\left(L_{\varkappa}^{1}\left(\mathbb{R}^{+}\right) ; A\right)$ onto $W_{\varkappa}(A)$. It remains to prove that $T \in \operatorname{Hom}\left(L_{\varkappa}^{1}\left(\mathbb{R}^{+}\right) ; A\right)$ if 
and only if $T \varphi_{\bullet}$ is a pseudoresolvent. The "only if" part is (9.5). Now suppose that $T \in L\left(L_{\varkappa}^{1}\left(\mathbb{R}^{+}\right) ; A\right)$ and $T \phi \bullet$ is a pseudoresolvent. Let $\lambda \in(\omega, \infty)$, $\mu \in(\omega, \infty), \lambda \neq \mu$. Then

$$
T\left(\phi_{\lambda} * \phi_{\mu}\right)=T\left(\frac{1}{\mu-\lambda}\left[\phi_{\lambda}-\phi_{\mu}\right]\right)=\frac{1}{\mu-\lambda}\left[T \phi_{\lambda}-T \phi_{\mu}\right]
$$

because $\phi_{\bullet}$ is a pseudoresolvent, whence $T\left(\phi_{\lambda} * \phi_{\mu}\right)=T \phi_{\lambda} \cdot T \phi_{\mu}$, because $T \phi_{\bullet}$ is a pseudoresolvent. Thus, by continuity,

$$
T(\varphi * \psi)=T \varphi \cdot T \psi
$$

for all $\varphi$ and $\psi$ in $\left\{\phi_{\lambda}: \lambda \in(\omega, \infty)\right\}$. By bilinearity and density (Lemma $2.1)$, this remains true for all $\varphi$ and $\psi$ in $L_{\varkappa}^{1}\left(\mathbb{R}^{+}\right)$.

Corollary 9.2. Suppose that $C$ is a closed convex cone in a Banach algebra $A$ such that $C \cdot C \subset C$. Let $T \in \operatorname{Hom}\left(L_{\varkappa}^{1}\left(\mathbb{R}^{+}\right) ; A\right)$. Then $T \varphi \in C$ for every positive $\varphi \in L_{\varkappa}^{1}\left(\mathbb{R}^{+}\right)$if and only if $T \phi_{\lambda} \in C$ for every $\lambda \in(\omega, \infty)$.

Proof. If $T \phi_{\lambda} \in C$ for every $\lambda \in(\omega, \infty)$, then $\left(T \phi_{\lambda}\right)^{n} \in C$ for all $\lambda \in(\omega, \infty)$ and $n=1,2, \ldots$ because $C \cdot C \subset C$. By (9.2), it follows that the map $T \phi_{\bullet}:(\omega, \infty) \rightarrow A$ is $C$-completely monotonic. Hence, by Theorem 6.1, the operator $T$ is $C$-positive.

10. Representations of the convolution algebra $L_{\varkappa}^{1}\left(\mathbb{R}^{+}\right)$and one-parameter semigroups of operators. Let $X$ be a Banach space, and $L(X)$ the Banach algebra of continuous linear operators from $X$ into $X$. Let $L\left(L_{\varkappa}^{1}\left(\mathbb{R}^{+}\right) ; L(X)\right)$ be the Banach space of linear operators from $L_{\varkappa}^{1}\left(\mathbb{R}^{+}\right)$into $L(X)$ continuous with respect to the norm topologies of $L_{\varkappa}^{1}\left(\mathbb{R}^{+}\right)$and $L(X)$. By the uniform boundedness principle, a linear map $T: L_{\varkappa}^{1}\left(\mathbb{R}^{+}\right) \rightarrow L(X)$ belongs to $L\left(L_{\varkappa}^{1}\left(\mathbb{R}^{+}\right) ; L(X)\right)$ if and only if the bilinear map $L_{\varkappa}^{1}\left(\mathbb{R}^{+}\right) \times X \ni$ $(\varphi, x) \rightarrow T(\varphi) x \in X$ is continuous with respect to the norm topologies of $L_{\varkappa}^{1}\left(\mathbb{R}^{+}\right)$and $X$.

A continuous representation of the convolution algebra $L_{\varkappa}^{1}\left(\mathbb{R}^{+}\right)$on a Banach space $X$ is, by definition, an element of $\operatorname{Hom}\left(L_{\varkappa}^{1}\left(\mathbb{R}^{+}\right) ; L(X)\right)$, i.e. an operator $T$ in $L\left(L_{\varkappa}^{1}\left(\mathbb{R}^{+}\right) ; L(X)\right)$ such that $T(\varphi * \psi)=T(\varphi) \cdot T(\psi)$ for all $\varphi$ and $\psi$ in $L_{\varkappa}^{1}\left(\mathbb{R}^{+}\right)$.

According to Section 8.4, the convolution Banach algebra $L_{\varkappa}^{1}\left(\mathbb{R}^{+}\right)$has a bounded approximate unit. Hence the Cohen-Hewitt factorization theorem ([H-R;II], p. 268, Theorem 32.22; [Pal], p. 535, Theorem 5.2.2) implies that whenever $X$ is a Banach space and $T \in \operatorname{Hom}\left(L_{\varkappa}^{1}\left(\mathbb{R}^{+}\right) ; L(X)\right)$, then the set

$$
Y=T\left(L_{\varkappa}^{1}\left(\mathbb{R}^{+}\right)\right) X=\left\{T(\varphi) x: \varphi \in L_{\varkappa}^{1}\left(\mathbb{R}^{+}\right), x \in X\right\}
$$

is a closed linear subspace of $X$. Applying the same factorization theorem to $X=L_{\varkappa}^{1}\left(\mathbb{R}^{+}\right)$and to $\widetilde{T}: L_{\varkappa}^{1}\left(\mathbb{R}^{+}\right) \ni \varphi \rightarrow \varphi * \in L\left(L_{\varkappa}^{1}\left(\mathbb{R}^{+}\right)\right)$and making 
use of the fact that, by $(8.12), L_{\varkappa}^{1}\left(\mathbb{R}^{+}\right) * L_{\varkappa}^{1}\left(\mathbb{R}^{+}\right)$is dense in $L_{\varkappa}^{1}\left(\mathbb{R}^{+}\right)$, one concludes that $L_{\varkappa}^{1}\left(\mathbb{R}^{+}\right) * L_{\varkappa}^{1}\left(\mathbb{R}^{+}\right)=L_{\varkappa}^{1}\left(\mathbb{R}^{+}\right)$. Hence

$$
Y=T\left(L_{\varkappa}^{1}\left(\mathbb{R}^{+}\right)\right) Y=\left\{T(\varphi) y: \varphi \in L_{\varkappa}^{1}\left(\mathbb{R}^{+}\right), y \in Y\right\} .
$$

LEMMA 10.1. Let $T$ be a continuous representation of the convolution algebra $L_{\varkappa}^{1}\left(\mathbb{R}^{+}\right)$on a Banach space $X$, and let $Y$ be defined by (10.1). Then for every $t \in \overline{\mathbb{R}^{+}}$and $y \in Y$ the derivative $\frac{d}{d t}\left[T\left(1_{(0, t]}\right) y\right]$ exists in the norm topology of $X$. Furthermore, whenever

$$
y=T(\varphi) x,
$$

where $\varphi \in L_{\varkappa}^{1}\left(\mathbb{R}^{+}\right)$and $x \in X$, then

$$
\frac{d}{d t}\left[T\left(1_{(0, t]}\right) y\right]=T\left(U_{t} \varphi\right) x
$$

for every $t \in \overline{\mathbb{R}^{+}}$, where $U_{t} \varphi$ is the right translate of $\varphi$ by $t$.

Proof. As a consequence of (8.10), if $\varphi \in L_{\varkappa}^{1}\left(\mathbb{R}^{+}\right)$and $t \in \overline{\mathbb{R}^{+}}$, then $T\left(U_{t} \varphi\right)=\frac{d}{d t}\left[T\left(1_{(0, t]}\right) T(\varphi)\right]$, the derivative being computed in the norm of $L(X)$. Hence, if $t \in \overline{\mathbb{R}^{+}}, y \in Y, \varphi \in L_{\varkappa}^{1}\left(\mathbb{R}^{+}\right), x \in X$, and (10.3) is satisfied, then (10.4) holds with the derivative computed in the norm of $X$.

THEOREM 10.2. Let $T$ be a continuous representation of the convolution algebra $L_{\varkappa}^{1}\left(\mathbb{R}^{+}\right)$on a Banach space $X$, and let $Y$ be the closed linear subspace of $X$ defined by (10.1). Let $\left(U_{t}\right)_{t \in \overline{\mathbb{R}^{+}}}$be the semigroup of right translations in $L_{\varkappa}^{1}\left(\mathbb{R}^{+}\right)$. Then there is a unique $C_{0}$ semigroup $\left(S_{t}\right)_{t \in \overline{\mathbb{R}^{+}}} \subset L(Y)$ such that

$$
S_{t} T(\varphi)=T\left(U_{t} \varphi\right) \quad \text { for every } t \in \overline{\mathbb{R}^{+}} \text {and } \varphi \in L_{\varkappa}^{1}\left(\mathbb{R}^{+}\right) .
$$

This unique semigroup satisfies the estimate

$$
\left\|S_{t}\right\|_{L(Y)} \leq \varkappa(t)\|T\|_{L\left(L_{\varkappa}^{1}\left(\mathbb{R}^{+}\right) ; L(X)\right)} \quad \text { for every } t \in \mathbb{R}^{+} .
$$

Proof. Uniqueness. If $t \in \overline{\mathbb{R}^{+}}, S_{t} \in L(Y)$ and $S_{t} T(\varphi)=T\left(U_{t} \varphi\right)$ for every $\varphi \in L_{\varkappa}^{1}\left(\mathbb{R}^{+}\right)$, then $S_{t} y=\frac{d}{d t}\left[T\left(1_{(0, t]}\right) y\right]$ for every $y \in Y$, by Lemma 10.1.

Existence. By Lemma 10.1, for every $t \in \overline{\mathbb{R}^{+}}$there is an algebraically linear operator $S_{t}: Y \rightarrow Y$ such that

$$
S_{t} y=\frac{d}{d t}\left[T\left(1_{(0, t]}\right) y\right] \quad \text { for every } y \in Y,
$$

the derivative being computed in the norm topology of $X$. It follows that $\left\|S_{t} y\right\|=\lim _{h \downarrow 0} \frac{1}{h}\left\|T\left(1_{(t, t+h]}\right) y\right\| \leq\|T\|\left(\lim _{h \downarrow 0} \frac{1}{h}\left\|1_{(t, t+h]}\right\|_{1, \varkappa}\right)\|y\|=\|T\| \varkappa(t)\|y\|$, so that $S_{t} \in L(Y)$ and $\left\|S_{t}\right\|_{L(Y)} \leq\|T\|_{L\left(L_{\varkappa}^{1}\left(\mathbb{R}^{+}\right) ; L(X)\right)} \cdot \varkappa(t)$. Again from (10.7), and from Lemma 10.1, it follows that $S_{t} T(\varphi) x=T\left(U_{t} \varphi\right) x$ for all $\varphi \in L_{\varkappa}^{1}\left(\mathbb{R}^{+}\right)$and $x \in X$. Hence $\left(S_{t}\right)_{t \in \overline{\mathbb{R}^{+}}} \subset L(Y)$ satisfies (10.5) and (10.6). 
Since every $y \in Y$ may be represented in the form (10.3), from (10.5) it follows that $S_{0}=\left.\mathrm{id}\right|_{Y}$ and for every $y \in Y$ the $Y$-valued function $t \rightarrow S_{t} y$ is continuous on $\overline{\mathbb{R}^{+}}$in the norm topology of $Y$. Finally, whenever $t_{1} \in \overline{\mathbb{R}^{+}}$, $t_{2} \in \overline{\mathbb{R}^{+}}, y \in Y$, and $\varphi \in L_{\varkappa}^{1}\left(\mathbb{R}^{+}\right)$and $x \in X$ are chosen to satisfy (10.3), then, by $(10.5)$,

$$
\begin{aligned}
S_{t_{1}} S_{t_{2}} y & =S_{t_{1}} S_{t_{2}} T(\varphi) x=S_{t_{1}} T\left(U_{t_{2}} \varphi\right) x=T\left(U_{t_{1}} U_{t_{2}} \varphi\right) x \\
& =T\left(U_{t_{1}+t_{2}} \varphi\right) x=S_{t_{1}+t_{2}} T(\varphi) x=S_{t_{1}+t_{2}} y .
\end{aligned}
$$

Thus $\left(S_{t}\right)_{t \in \overline{\mathbb{R}^{+}}}$is a $C_{0}$ semigroup.

Remarks. Without using the Cohen-Hewitt factorization theorem, one could define $Y=\overline{\operatorname{span}} T\left(L_{\varkappa}^{1}\left(\mathbb{R}^{+}\right)\right) X$ and prove Theorem 10.2 by an almost unchanged argument. According to W. Chojnacki $[\mathrm{C}]$, the semigroup appearing in Theorem 10.2 may by defined by $S_{t}=\widetilde{\widetilde{T}}\left(\varepsilon_{t}\right)$ where $\varepsilon_{t}$ is the unit mass at $t, \widetilde{T}=\widetilde{T} W, \widetilde{T}$ is the extension (of B. E. Johnson's type) of $\left.T\right|_{Y}$ to a representation on $Y$ of the algebra $\mathcal{D}\left(L_{\varkappa}^{1}\left(\mathbb{R}^{+}\right)\right)$of centralizers of $L_{\varkappa}^{1}\left(\mathbb{R}^{+}\right)$and $W$ is an isomorphism (of J. G. Wendel's type) of the convolution algebra $\mathcal{M}_{\varkappa}(K)$ onto $\mathcal{D}\left(L_{\varkappa}^{1}\left(\mathbb{R}^{+}\right)\right)$. Here $\mathcal{M}_{\varkappa}(K)$ is the set of signed (if $K=\mathbb{R}$ ) or complex (if $K=\mathbb{C}$ ) measures $\mu$ on $\overline{\mathbb{R}^{+}}$such that $\|\mu\|_{\mathcal{M}_{\varkappa}}=\int_{\overline{\mathbb{R}^{+}}} \varkappa(t)|\mu|(d t)<\infty$. Such an approach to Theorem 10.2 follows the familiar method of reconstructing a representation of a locally compact group $G$ from a representation of the group algebra $L^{1}(G)$. See $[J]$, Sec. 9; [H-R;I], Theorem 22.7; [Pal], Sec. 1.9.13.

COROLlary 10.3. Under the assumptions of Theorem 10.2,

$$
T(\varphi) T(\psi)=T(\psi) T(\varphi)=\int_{0}^{\infty} \varphi(t) S_{t} T(\psi) d t
$$

for all $\varphi$ and $\psi$ in $L_{\varkappa}^{1}\left(\mathbb{R}^{+}\right)$, the integral of the $L(X ; Y)$-valued function $t \rightarrow \varphi(t) S_{t} T(\psi)=\varphi(t) T\left(U_{t} \psi\right)$ being taken in the sense of Bochner. As a consequence,

$$
T(\varphi)=\lim _{\lambda \rightarrow \infty} T\left(\lambda \phi_{\lambda} * \varphi\right)=\lim _{\lambda \rightarrow \infty} \int_{0}^{\infty} \varphi(t) S_{t} T\left(\lambda \phi_{\lambda}\right) d t
$$

for every $\varphi \in L_{\varkappa}^{1}\left(\mathbb{R}^{+}\right)$, the limit being taken in the norm topology of $L(X)$, and

$$
T(\varphi) y=\int_{0}^{\infty} \varphi(t) S_{t} y d y
$$

for all $\varphi \in L_{\varkappa}^{1}\left(\mathbb{R}^{+}\right)$and $y \in Y$, the integral of the $Y$-valued function $t \rightarrow$ $\varphi(t) S_{t} y$ being taken in the sense of Bochner. 
Pr o of. According to Lemma 8.3.1 and (10.5),

$$
\begin{aligned}
T(\varphi) T(\psi) & =T(\varphi * \psi)=T\left(\int_{0}^{\infty} \varphi(t) U_{t} \psi d t\right) \\
& =\int_{0}^{\infty} \varphi(t) T\left(U_{t} \psi\right) d t=\int_{0}^{\infty} \varphi(t) S_{t} T(\psi) d t
\end{aligned}
$$

for all $\varphi$ and $\psi$ in $L_{\varkappa}^{1}\left(\mathbb{R}^{+}\right)$, proving (10.8). The equality (10.9) follows from (8.12) and (10.8), while (10.10) follows from (10.8) by representing $y$ in the form $y=T(\psi) x$.

11. Pseudoresolvents on $(\omega, \infty)$ with values in $L(X)$, their regularity spaces, and their generators. The material of the present chapter forms the basis for Theorem 12.5. However for Theorem 12.2 this material is unnecessary.

Let $X$ be a Banach space, and $L(X)$ the Banach algebra of continuous linear operators on $X$. We will consider a pseudoresolvent on $(\omega, \infty)$ with values in $L(X)$, i.e. a map

$$
(\omega, \infty) \ni \lambda \rightarrow R_{\lambda} \in L(X)
$$

satisfying the resolvent equation

$$
R_{\lambda}-R_{\mu}=(\mu-\lambda) R_{\lambda} R_{\mu}
$$

for all $\lambda$ and $\mu$ in $(\omega, \infty)$. It follows from (11.2) that $\left(R_{\lambda}\right)_{\lambda \in(\omega, \infty)}$ is a commutative family of operators, and that the kernel $K$ and range $\Im$ of $R_{\lambda}$ are both independent of $\lambda$. See [Y;II], pp. 215-216. The formula

$$
G=\left\{(x, y) \in X \times X: \lambda R_{\lambda} x-x=R_{\lambda} y \text { for every } \lambda \in(\omega, \infty)\right\}
$$

defines a closed linear subspace of $X \times X$. Following [D-M; XII-XVI], p. 243, we will call $G$ the extended generator of the pseudoresolvent (11.1). Equation (11.2) implies that

(11.4) if $x \in X, y \in X$, and there exists a $\mu \in(\omega, \infty)$ such that $\mu R_{\mu} x-x$ $=R_{\mu} y$, then $(x, y) \in G$.

Indeed, it follows from (11.2) that if $\mu R_{\mu} x-x=R_{\mu} y$, then

$$
\begin{aligned}
R_{\lambda} y & =\left[1+(\mu-\lambda) R_{\lambda}\right] R_{\mu} y=\left[1+(\mu-\lambda) R_{\lambda}\right]\left[\mu R_{\mu} x-x\right] \\
& =\mu R_{\mu} x-x+\mu(\mu-\lambda) R_{\lambda} R_{\mu} x+(\lambda-\mu) R_{\lambda} x \\
& =\mu R_{\mu} x-x+\mu\left(R_{\lambda}-R_{\mu}\right) x+(\lambda-\mu) R_{\lambda} x=\lambda R_{\lambda} x-x
\end{aligned}
$$

for every $\lambda \in(\omega, \infty)$. The domain of the extended generator $G$ is, by definition, the set

$$
D(G)=\{x \in X: \text { there exists } y \in X \text { such that }(x, y) \in G\} .
$$


It follows that

$$
D(G)=\Im \text {. }
$$

Indeed, if $(x, y) \in G$ and $\mu \in(\omega, \infty)$, then $x=R_{\mu}(\mu x-y) \in \Im$. Conversely, if $x \in \Im$ and $\mu \in(\omega, \infty)$, then $x=R_{\mu} z$ for some $z \in X$, so that $\mu R_{\mu} x-x=$ $R_{\mu} y$ for $y=\mu x-z$, whence $(x, y) \in G$ by virtue of (11.4).

Appendix I in $[\mathrm{Ki}]$ contains a necessary and sufficient condition for a subspace of $X \times X$ to be the extended generator of a pseudoresolvent. If a pseudoresolvent (11.1) is the Laplace transform of a measurable contraction semigroup in a function space, then the extended generator (11.3) coincides with the full generator of the semigroup defined in [E-K], pp. 23-24. See also [R-Y], p. 263.

If $K=\{0\}$ then $G$ is the graph of a closed operator from $X$ into $X$ whose resolvent set contains $(\omega, \infty)$, and the pseudoresolvent (11.1) is the resolvent of this operator.

According to [D-M;C], p. 314, the regularity space of the pseudoresolvent (11.1) is, by definition, the linear set

$$
\mathfrak{R}=\left\{x \in X: \lim _{\lambda \rightarrow \infty}\left\|\lambda R_{\lambda} x-x\right\|=0\right\} .
$$

It is obvious that

$$
\Re \subset \bar{\Im},
$$

where $\bar{\Im}$ denotes the closure of $\Im$ in the norm topology of $X$. If $x \in \mathfrak{R} \cap K$, then $x=\lim _{\lambda \rightarrow \infty} \lambda R_{\lambda} x=\lim _{\lambda \rightarrow \infty} \lambda \cdot 0=0$, so that

$$
\mathfrak{R} \cap K=\{0\} .
$$

From commutativity of the family of operators $\left(R_{\lambda}\right)$, it follows that

$$
R_{\lambda} \mathfrak{R} \subset \mathfrak{R}
$$

for every $\lambda \in(\omega, \infty)$.

According to [Hi], p. 98, and [D-M;C], p. 314, the generator of the pseudoresolvent (11.1) is defined to be the operator $A$ from $X$ into $X$ with domain $D(A)$ such that

(11.11) $x \in D(A)$ and $y=A x$ if and only if $\lim _{\lambda \rightarrow \infty}\left\|\lambda\left(\lambda R_{\lambda} x-x\right)-y\right\|=0$.

Denote by $G(A)$ the graph of $A$. Definition (11.11) is equivalent to

$$
G(A)=\left\{(x, y) \in X \times X: \lim _{\lambda \rightarrow \infty}\left\|\lambda\left(\lambda R_{\lambda} x-x\right)-y\right\|=0\right\} .
$$

It is obvious that

$$
D(A) \subset \Re .
$$

Furthermore,

$$
(X \times \Re) \cap G \subset G(A) \subset(\mathfrak{R} \times \bar{\Im}) \cap G .
$$


Indeed, if $(x, y) \in(X \times \mathfrak{R}) \cap G$, then $\lim _{\lambda \rightarrow \infty} \lambda R_{\lambda} y=y$ and $\lambda R_{\lambda} x-x=R_{\lambda} y$ for every $\lambda \in(\omega, \infty)$, so that $y=\lim _{\lambda \rightarrow \infty} \lambda\left(\lambda R_{\lambda} x-x\right)$ and $(x, y) \in G(A)$. Hence $(X \times \mathfrak{R}) \cap G \subset G(A)$. If $(x, y) \in G(A)$, then $x \in \mathfrak{R} \subset \bar{\Im}$, by (11.13) and (11.8), so that $\lambda\left(\lambda R_{\lambda} x-x\right) \in \bar{\Im}$ for every $\lambda \in(\omega, \infty)$, and hence $y=\lim _{\lambda \rightarrow \infty} \lambda\left(\lambda R_{\lambda} x-x\right) \in \bar{\Im}$. Thus $G(A) \subset \mathfrak{R} \times \bar{\Im}$. Furthermore, if $(x, y) \in$ $G(A)$ and $\mu \in(\omega, \infty)$, then

$$
\begin{aligned}
R_{\mu} y & =R_{\mu} \lim _{\lambda \rightarrow \infty} \lambda\left(\lambda R_{\lambda} x-x\right)=\lim _{\lambda \rightarrow \infty} \lambda\left(\lambda R_{\mu} R_{\lambda} x-R_{\mu} x\right) \\
& =\lim _{\lambda \rightarrow \infty} \lambda\left(\mu R_{\mu} R_{\lambda} x-R_{\lambda} x\right)=\left(\mu R_{\mu}-1\right) \lim _{\lambda \rightarrow \infty} \lambda R_{\lambda} x=\mu R_{\mu} x-x,
\end{aligned}
$$

by (11.2) and (11.13). Hence $G(A) \subset(\Re \times \bar{\Im}) \cap G$.

EXAmple. Consider an operator $B \in L(X)$ such that $B^{2}=0$. The constant map $(\omega, \infty) \ni \lambda \rightarrow B \in L(X)$ is then a pseudoresolvent for which $\Im \subset K, G=\{(-B y, y): y \in X\}$ and $\mathfrak{R}=\{0\}$.

From now on we will make some additional assumptions on the pseudoresolvent (11.1). The statements of Lemma 11.1 to Proposition 11.4 are contained in Section 7, pp. 314-316, of Chapter XII of [D-M;C]. Our presentation expounds the role of the extended generator $G$.

Lemma 11.1. If $\lim _{\lambda \rightarrow \infty}\left\|R_{\lambda} x\right\|=0$ for every $x \in X$, then $\Im \subset \mathfrak{R}$.

Proof. Let $x \in \Im$. Fix $\mu \in \mathbb{R}^{+}$and choose $z \in X$ such that $x=R_{\mu} z$. Then, by (11.2), $\lambda R_{\lambda} x-x=\lambda R_{\lambda} R_{\mu} z-R_{\mu} z=R_{\lambda}\left(\mu R_{\mu} z-z\right)$, so that $x \in \mathfrak{R}$.

Proposition 11.2. If

$$
\limsup _{\lambda \rightarrow \infty} \lambda\left\|R_{\lambda}\right\|_{L(X)}<\infty,
$$

then

$$
\Re=\bar{\Im}
$$

and

$$
G(A)=(X \times \mathfrak{R}) \cap G=(\mathfrak{R} \times \mathfrak{R}) \cap G .
$$

Proof. Condition (11.15) implies that $\bar{\Re}=\mathfrak{R}$, and hence equalities (11.16) and (11.17) follow from (11.8), (11.14) and Lemma 11.1.

Corollary 11.3. If condition (11.15) is satisfied then $\mathfrak{R}$ is a closed linear subspace of $X$, and $A$ is a closed operator from $X$ into $X$ with domain and range contained in $\mathfrak{R}$.

The following Proposition 11.4 implies at once that a pseudoresolvent which has range dense in $X$ and satisfies (11.15) is the resolvent of a densely defined operator. See $[\mathrm{D}]$, p. 37, Theorem 2.6; [P], p. 37, Theorem 9.4. 
Proposition 11.4. If condition (11.15) is satisfied and $A$ is treated as an operator from $\mathfrak{R}$ into $\mathfrak{R}$ then the resolvent set of $A$ contains $(\omega, \infty)$ and

$$
(\lambda-A)^{-1}=\left.R_{\lambda}\right|_{\Re}
$$

for every $\lambda \in(\omega, \infty)$. Furthermore, $D(A)$ is dense in $\mathfrak{R}$.

Proof. If $x \in D(A)$ and $\lambda \in(\omega, \infty)$, then $(x, A x) \in G$ by (11.14), whence $\lambda R_{\lambda} x-x=R_{\lambda} A x$. This means that

$$
R_{\lambda}(\lambda-A) x=x \quad \text { for all } \lambda \in(\omega, \infty) \text { and } x \in D(A) .
$$

If $x \in \mathfrak{R}$ and $\lambda \in(\omega, \infty)$, then, by Proposition 11.2, $R_{\lambda} x \in \Im \subset \mathfrak{R}$ and $\left(R_{\lambda} x, \lambda R_{\lambda} x-x\right) \in(\mathfrak{R} \times \mathfrak{R}) \cap G=G(A)$, so that $R_{\lambda} x \in D(A)$ and $(\lambda-A) R_{\lambda} x=\lambda R_{\lambda} x-A R_{\lambda} x=\lambda R_{\lambda} x-\left[\lambda R_{\lambda} x-x\right]=x$. Hence

(11.20) $\quad R_{\lambda} x \in D(A)$ and $(\lambda-A) R_{\lambda} x=x \quad$ for all $\lambda \in(\omega, \infty)$ and $x \in \mathfrak{R}$.

From (11.19), (11.20) and Corollary 11.3 it follows that if $A$ is treated as an operator from $\mathfrak{R}$ into $\mathfrak{R}$, then its resolvent set contains $(\omega, \infty)$ and $(11.18)$ holds. Furthermore, by (11.20), if $x \in \mathfrak{R}$ then $\lambda R_{\lambda} x \in D(A)$ for every $\lambda \in(\omega, \infty)$ and hence $x=\lim _{\lambda \rightarrow \infty} \lambda R_{\lambda} x$ belongs to the closure of $D(A)$, proving that $D(A)$ is dense in $\Re$.

Proposition 11.5. If the Banach space $X$ is reflexive and the pseudoresolvent (11.1) satisfies condition (11.15), then $X$ splits into the direct sum $X=K+\mathfrak{R}$.

Pr o of. Since every reflexive Banach space is locally sequentially weakly compact, Proposition 11.5 is a consequence of Corollary $1^{\prime}$ in [Y;II], Chap. VIII, Sec. 4, p. 218. The following proof does not refer to weak compactness. The direct sum $K+\mathfrak{R}$ is a closed subspace of $X$, because the projector $P$ of $K+\mathfrak{R}$ onto $\mathfrak{R}$ witk $\operatorname{Ker}(P)=K$ is continuous. Indeed, $P x=\lim _{\lambda \rightarrow \infty} \lambda R_{\lambda} x$ for every $x \in K+\mathfrak{R}$, and continuity of $P$ follows from (11.15). Let $X^{*}$ be the space adjoint to $X$, and for every $\lambda \in(\omega, \infty)$ let $R_{\lambda}^{*}$ be the operator adjoint to $R_{\lambda}$. The map

$$
(\omega, \infty) \ni \lambda \rightarrow R_{\lambda}^{*} \in L\left(X^{*}\right)
$$

is a pseudoresolvent satisfying a condition analogous to (11.15). Let $K^{*}=$ Ker $R_{\lambda}^{*}, \Im^{*}=$ Range $R_{\lambda}^{*}$, and let $\mathfrak{R}^{*}$ be the regularity space of the pseudoresolvent (11.21). Then $K^{*} \cap \mathfrak{R}^{*}=\{0\}$ and, by Proposition 11.2, $R^{*}=\overline{\Im^{*}}$. Furthermore, $\mathfrak{R}^{\perp}=\Im^{\perp}=K^{*}$ and ${ }^{\perp} \mathfrak{R}^{*}={ }^{\perp} \Im^{*}=K$. If $X$ is reflexive, then $K^{\perp}=\left({ }^{\perp} \mathfrak{R}^{*}\right)^{\perp}=\mathfrak{R}^{*}$, by Theorem 2 in [Y;II], p. 137. As a consequence, $(K+\mathfrak{R})^{\perp}=K^{\perp} \cap \mathfrak{R}^{\perp}=\mathfrak{R}^{*} \cap K^{*}=\{0\}$ and $K+\mathfrak{R}=X$.

12. The Hille-Yosida theorem. Let $X$ be a Banach space, and $L(X)$ the Banach algebra of continuous linear operators on $X$. The following Proposition 12.1 coincides with Theorem 11 in Sec. VIII.1 of [D-S;I]. 
Proposition 12.1. Let $\left(S_{t}\right)_{t \in \overline{\mathbb{R}^{+}}} \subset L(X)$ be a $C_{0}$ semigroup such that $\sup _{t \in \overline{\mathbb{R}^{+}}}(\varkappa(t))^{-1}\left\|S_{t}\right\|_{L(X)}<\infty$. Let $A$ be a linear operator from $X$ into $X$ with domain $D(A)$. Then the following two conditions are equivalent:

(a) $A$ is the infinitesimal generator of the semigroup $\left(S_{t}\right)_{t \in \overline{\mathbb{R}^{+}}}$, i.e. the graph of $A$ is equal to

$$
\left\{(x, y) \in X \times X: \lim _{h \downarrow 0}\left\|\frac{1}{h}\left(S_{h} x-x\right)-y\right\|_{X}=0\right\},
$$

(b) $A$ is a closed operator from $X$ into $X$, the resolvent set of $A$ contains $(\omega, \infty)$, and

$$
(\lambda-A)^{-1} x=\int_{0}^{\infty} e^{-\lambda t} S_{t} x d t \quad \text { for all } \lambda \in(\omega, \infty) \text { and } x \in X .
$$

P r o o f. We follow [D-S;I]. There is at most one operator $A$ satisfying (b), and hence we are reduced to proving $(\mathrm{a}) \Rightarrow(\mathrm{b})$. So, suppose that (a) holds. Note that

$$
\begin{aligned}
\frac{1}{h}\left(S_{h}-1\right) \int_{0}^{\infty} e^{-\lambda t} S_{t} x d t & =\int_{0}^{\infty} e^{-\lambda t} S_{t} \frac{1}{h}\left(S_{h}-1\right) x d t \\
& =\frac{e^{\lambda h}-1}{h} \int_{0}^{\infty} e^{-\lambda t} S_{t} x d t-e^{\lambda h} \frac{1}{h} \int_{0}^{h} e^{-\lambda t} S_{t} x d t
\end{aligned}
$$

for all $h \in \mathbb{R}^{+}, \lambda \in(\omega, \infty)$ and $x \in X$. By a passage to the limit as $h \downarrow 0$, it follows that

$$
\int_{0}^{\infty} e^{-\lambda t} S_{t}(\lambda-A) x d t=x \quad \text { for all } \lambda \in(\omega, \infty) \text { and } x \in D(A),
$$

and

$$
\begin{array}{r}
\int_{0}^{\infty} e^{-\lambda t} S_{t} x d t \in D(A) \text { and } \quad(\lambda-A) \int_{0}^{\infty} e^{-\lambda t} S_{t} x d t=x \\
\text { for all } \lambda \in(\omega, \infty) \text { and } x \in X .
\end{array}
$$

These equalities mean that for every $\lambda \in(\omega, \infty)$ the operator $\lambda-A$, defined on $D(A)$, has inverse $(\lambda-A)^{-1}$ defined on the whole $X$ and $(\lambda-A)^{-1} x=$ $\int_{0}^{\infty} e^{-\lambda t} S_{t} x d t$ for every $x \in X$. It follows that $A$ is closed and its resolvent set contains $(\omega, \infty)$.

The following Theorem 12.2 refines the Hille-Yosida generation theorem in the sense of extending the class of weight functions considered from those of the form $\varkappa(t)=e^{\omega t}$ to those described in Section 1. However both the theorems apply to the same class of semigroups, namely to all $C_{0}$ semigroups. 
Theorem 12.2. Let $A$ be a linear operator from a Banach space $X$ into $X$ with domain $D(A)$. Then the following two conditions are equivalent:

(i) $A$ is the infinitesimal generator of a $C_{0}$ semigroup $\left(S_{t}\right)_{t \in \overline{\mathbb{R}^{+}}} \subset L(X)$ such that $\sup _{t \in \overline{\mathbb{R}^{+}}}(\varkappa(t))^{-1}\left\|S_{t}\right\|_{L(X)}<\infty$.

(ii) $D(A)$ is dense in $X, A$ is a closed operator from $X$ into $X$ with resolvent set containing $(\omega, \infty)$, and the map $(\omega, \infty) \ni \lambda \rightarrow(\lambda-A)^{-1} \in L(X)$ belongs to the Widder space $W_{\varkappa}(L(X))$.

If the above equivalent conditions are satisfied, then

(iii) $\sup _{t \in \mathbb{R}^{+}}(\varkappa(t))^{-1}\left\|S_{t}\right\|_{L(X)}=\sup _{\lambda \in(\omega, \infty), n=1,2, \ldots} \frac{\left\|(\lambda-A)^{-n}\right\|_{L(X)}}{\left\|\phi_{\lambda}^{* n}\right\|_{1, \varkappa}}$.

Proof. (i) $\Rightarrow[($ ii) \&(iii)]. It is easy to check that if (i) holds, then there is a unique continuous representation $T$ of the convolution algebra $L_{\varkappa}^{1}\left(\mathbb{R}^{+}\right)$ on the Banach space $X$ such that

$$
T(\varphi) x=\int_{0}^{\infty} \varphi(t) S_{t} x d t \quad \text { for all } \varphi \in L_{\varkappa}^{1}\left(\mathbb{R}^{+}\right) \text {and } x \in X,
$$

the integral being understood in the sense of Bochner. It is also easy to prove that

$$
\|T\|_{L\left(L_{\varkappa}^{1}\left(\mathbb{R}^{+}\right) ; L(X)\right)}=\sup _{t \in \mathbb{R}^{+}}(\varkappa(t))^{-1}\left\|S_{t}\right\|_{L(X)} .
$$

From (12.1) it follows that the set $T\left(C_{\mathrm{c}}^{1}\left(\mathbb{R}^{+}\right)\right) X$ is dense in $X$, and that $T\left(C_{\mathrm{c}}^{1}\left(\mathbb{R}^{+}\right)\right) X \subset D(A)$. Hence $D(A)$ is dense in $X$.

According to Theorem 9.1, the map $T \phi_{\bullet}$ is a pseudoresolvent in $W_{\varkappa}(L(X))$ such that

$$
\left\|T \phi_{\bullet}\right\|_{W_{\varkappa}(L(X))}=\|T\|_{L\left(L_{\varkappa}^{1}\left(\mathbb{R}^{+}\right) ; L(X)\right)} .
$$

By Proposition 12.1 and (12.1), $A$ is a closed operator from $X$ into $X$, the resolvent set of $A$ contains $(\omega, \infty)$, and $(\lambda-A)^{-1}=T \phi_{\lambda}$ for every $\lambda \in(\omega, \infty)$. The last equality implies that the map $(\omega, \infty) \ni \lambda \rightarrow(\lambda-A)^{-1}$ $\in L(X)$ belongs to $W_{\varkappa}(L(X))$. Finally, (iii) follows from (9.4), (12.2) and (12.3).

(ii) $\Rightarrow$ (i). Suppose that (ii) holds. Then the map $(\omega, \infty) \ni \lambda \rightarrow(\lambda-A)^{-1}$ $\in L(X)$ is a pseudoresolvent in $W_{\varkappa}(L(X))$. By Theorem 9.1 there is a $T \in \operatorname{Hom}\left(L_{\varkappa}^{1}\left(\mathbb{R}^{+}\right) ; L(X)\right)$ such that

$$
T\left(\phi_{\lambda}\right)=(\lambda-A)^{-1} \quad \text { for every } \lambda \in(\omega, \infty) .
$$

It follows that $D(A) \subset Y$, where, according to the notation of Chapter 10,

$$
Y=T\left(L_{\varkappa}^{1}\left(\mathbb{R}^{+}\right)\right) X \text {. }
$$

Since $D(A)$ is dense in $X$, the Cohen-Hewitt factorization theorem (see (10.1)) implies that $Y=X$. Thus, by Theorem 10.2, there is a unique $C_{0}$ 
semigroup $\left(S_{t}\right)_{t \in \overline{\mathbb{R}}^{+}} \subset L(X)$ satisfying (10.5) such that

$$
\sup _{t \in \overline{\mathbb{R}^{+}}}(\varkappa(t))^{-1}\left\|S_{t}\right\|_{L(X)}<\infty .
$$

By (10.10),

$$
(\lambda-A)^{-1} x=T\left(\phi_{\lambda}\right) x=\int_{0}^{\infty} e^{-\lambda t} S_{t} x d t \quad \text { for all } \lambda \in(\omega, \infty) \text { and } x \in X .
$$

Hence, by Proposition 12.1, $A$ is the infinitesimal generator of $\left(S_{t}\right)_{t \in \overline{\mathbb{R}}^{+}}$.

REMARK 12.3. If $X$ is a reflexive Banach space, then the postulate in (ii) that $D(A)$ is dense in $X$ is a consequence of the remaining part of this condition. Indeed, if $X$ is reflexive and $A$ is a closed operator from $X$ into $X$ with domain $D(A)$ such that the resolvent set of $A$ contains $(\omega, \infty)$ and $\lim \sup _{\lambda \rightarrow \infty} \lambda\left\|(\lambda-A)^{-1}\right\|<\infty$, then, according to T. Kato $[\mathrm{K} ; \mathrm{I}], D(A)$ is dense in $X$. This may be deduced from Proposition 11.5: for the pseudoresolvent $(\omega, \infty) \ni \lambda \rightarrow(\lambda-A)^{-1} \in L(X)$ one has $K=\{0\}$ and $\Im=D(A)$, whence $\overline{D(A)}=\bar{\Im}=\mathfrak{R}=\mathfrak{R}+K=X$.

Remark 12.4 ([E-K], Corollary 2.8(a), p. 14). Suppose that the equivalent conditions (i) and (ii) of Theorem 12.2 are satisfied and that $C$ is a closed convex cone in $X$. Then

$$
S_{t} C \subset C \quad \text { for every } t \in \overline{\mathbb{R}^{+}}
$$

if and only if

$$
(\lambda-A)^{-1} C \subset C \quad \text { for every } \lambda \in(\omega, \infty) .
$$

Indeed, (I) $\Rightarrow(\mathrm{II})$ is a simple consequence of Proposition 12.1. To prove (II) $\Rightarrow(\mathrm{I})$, let $T \in \operatorname{Hom}\left(L_{\varkappa}^{1}\left(\mathbb{R}^{+}\right) ; L(X)\right)$ be defined by $(12.1)$. Then $T\left(\phi_{\lambda}\right)=$ $(\lambda-A)^{-1}$ for every $\lambda \in(\omega, \infty)$, by Proposition 12.1(b). Hence, by Corollary 9.2 applied to the closed convex cone $\widetilde{C}=\{B \in L(X): B C \subset C\}$ in $L(X)$, condition (II) implies that $\int_{0}^{\infty} \varphi(t) S_{t} x d t=T(\varphi) x \in C$ whenever $x \in C$ and $\varphi \in L_{\varkappa}^{1}\left(\mathbb{R}^{+}\right)$is positive. From this (i) follows by an approximation $S_{t} x=\lim _{n \rightarrow \infty} \int_{0}^{\infty} \varphi_{n}(u) S_{u} x d u$ with positive $\varphi_{n} \in L_{\varkappa}^{1}\left(\mathbb{R}^{+}\right)$.

Theorem 12.5. Let $X$ be a Banach space and let $R_{\bullet}:(\omega, \infty) \ni \lambda \rightarrow$ $R_{\lambda} \in L(X)$ be a pseudoresolvent belonging to $W_{\varkappa}(L(X))$. Let $A$ be the generator of this pseudoresolvent (defined by (11.11)), $\mathfrak{R}$ its regularity space (defined by (11.7)), and $\Im$ the range of $R_{\lambda}$ (independent of $\lambda$ ). Then

(i) there is a unique representation $T$ of the convolution algebra $L_{\varkappa}^{1}\left(\mathbb{R}^{+}\right)$ on the Banach space $X$ such that $\|T\|_{L\left(L_{\varkappa}^{1}\left(\mathbb{R}^{+}\right) ; L(X)\right)}=\left\|R_{\bullet}\right\|_{W_{\varkappa}(L(X))}$ and $T \phi_{\bullet}=R_{\bullet}$,

(ii) $\mathfrak{R}=\bar{\Im}=T\left(L_{\varkappa}^{1}\left(\mathbb{R}^{+}\right)\right) X=T\left(L_{\varkappa}^{1}\left(\mathbb{R}^{+}\right)\right) \mathfrak{R}$, 
(iii) there is a unique $C_{0}$ semigroup $\left(S_{t}\right)_{t \in \mathbb{R}^{+}} \subset L(\Re)$ such that

$$
\left\|S_{t}\right\|_{L(\Re)} \leq \varkappa(t)\left\|R_{\bullet}\right\|_{W_{\varkappa}(L(X))} \quad \text { and } \quad S_{t} T(\varphi)=T\left(U_{t} \varphi\right)
$$

for all $t \in \overline{\mathbb{R}^{+}}$and $\varphi \in L_{\varkappa}^{1}\left(\mathbb{R}^{+}\right)$,

(iv) $\int_{0}^{\infty} e^{-\lambda t} S_{t} x d t=R_{\lambda} x$ for all $\lambda \in(\omega, \infty)$ and $x \in \mathfrak{R}$,

(v) $G(A) \subset \mathfrak{R} \times \mathfrak{R}$ and $A$ is a closed operator from $\mathfrak{R}$ into $\mathfrak{R}$,

(vi) if $A$ is treated as an operator from $\mathfrak{R}$ into $\mathfrak{R}$, then $(\omega, \infty)$ is contained in the resolvent set of $A,(\lambda-A)^{-1}=\left.R_{\lambda}\right|_{\mathfrak{R}}$ for every $\lambda \in(\omega, \infty)$, and $A$ is the infinitesimal generator of the semigroup in (iii).

Proof. Assertion (i) follows from Theorem 9.1. Since $R_{\bullet} \in W_{\varkappa}(L(X))$, from (2.3) and (9.4) it follows that $R_{\bullet}$ satisfies (11.15). Hence the equality $\mathfrak{R}=\bar{\Im}$ in (ii) follows from Proposition 11.2. From (i) and Lemma 2.1 it follows that, for every $\lambda \in(\omega, \infty), \Im=T\left(\phi_{\lambda}\right) X \subset T\left(L_{\varkappa}^{1}\left(\mathbb{R}^{+}\right)\right) X \subset \bar{\Im}$, and hence $T\left(L_{\varkappa}^{1}\left(\mathbb{R}^{+}\right)\right) X=\bar{\Im}$, by the Cohen-Hewitt factorization theorem. The last equality in (ii) follows from (10.2).

Assertions (iii) and (iv) follow from (i), (ii), Theorem 10.2, and (10.10). By (11.15) and Corollary 11.3, condition (v) holds. Furthermore, according to Proposition 11.4, if $A$ is treated as an operator from $\mathfrak{R}$ into $\mathfrak{R}$, then the resolvent set of $A$ contains $(\omega, \infty)$, and $(\lambda-A)^{-1}=\left.R_{\lambda}\right|_{\Re}$ for every $\lambda \in$ $(\omega, \infty)$. From the last equality, (iv), and Proposition 12.1, it follows that $A$ is the infinitesimal generator of the semigroup from (iii). Thus condition (vi) holds.

REMARK 12.5. It follows from Theorem 2.4 of [F-Y], p. 31, that the semigroup in Theorem 12.5(iii) extends to a semigroup $(\widetilde{S})_{t \in \overline{\mathbb{R}^{+}}} \subset L(\mathfrak{R}+K)$, $K=\operatorname{Ker} R_{\lambda}$, which admits on $\Re+K$ the Yosida approximation not necessarily uniform near $t=0$. (Recall that, according to Proposition 11.5, $\Re+K=X$ if $X$ is reflexive.) The operators $\left\langle e^{\bullet A}, \varphi\right\rangle$ and $e^{t A} * \varphi$ occurring on p. 33 of $[\mathrm{F}-\mathrm{Y}]$ are nothing but our $T(\varphi)$ and $T\left(U_{t} \check{\varphi}\right)$, where $\left[U_{t} \breve{\varphi}\right](s)=\varphi(t-s)$ if $s \in[0, t],=0$ if $s \in(t, \infty)$.

\section{References}

[A] W. Arendt, Vector-valued Laplace transforms and Cauchy problems, Israel J. Math. 59 (1987), 327-352.

[B] A. Bobrowski, On the Yosida approximation and the Widder-Arendt representation theorem, Studia Math. 124 (1997), 281-290.

[C] W. Chojnacki, Multiplier algebras, Banach bundles, and one-parameter semigroups, Ann. Scuola Norm. Sup. Pisa Cl. Sci. (4) 28 (1999), 287-322.

[D] E. B. Davies, One-Parameter Semigroups, Academic Press, 1980. 
[D-M;C] C. Dellacherie and P.-A. Meyer, Probabilities and Potential C. Potential Theory for Discrete and Continuous Semigroups, North-Holland Math. Stud. 151, North-Holland, 1988.

[D-M;XII-XVI] - - , Probabilités et Potentiel, Chapitres XII à XVI. Théorie du Potentiel Associée à une Résolvante, Théorie des Processus de Markov, Hermann, Paris, 1987.

[D-S;I] N. Dunford and J. T. Schwartz, Linear Operators, Part I, General Theory, Interscience, New York, 1958.

[D-S;II] -, - , Linear Operators, Part II. Spectral Theory, Self Adjoint Operators in Hilbert Space, Interscience, New York, 1963.

[D-U] J. Diestel and J. J. Uhl, Jr., Vector Measures, Math. Surveys 15, Amer. Math. Soc., Providence, RI, 1977.

[E-K] S. N. Ethier and T. G. Kurtz, Markov Processes, Characterization and Convergence, Wiley, 1986.

[F-Y] A. Favini and A. Yagi, Degenerate Differential Equations in Banach Spaces, Marcel Dekker, 1999.

[G] J. A. Goldstein, Semigroups of Linear Operators and Applications, Oxford Univ. Press, 1985.

[H] P. R. Halmos, Measure Theory, Springer, 1974

[H-N] B. Hennig and F. Neubrander, On representations, inversions, and approximations of Laplace transforms in Banach spaces, Appl. Anal. 49 (1993), 151-170.

[H-R;I] E. Hewitt and K. A. Ross, Abstract Harmonic Analysis, Volume I, Structure of Topological Groups, Integration Theory, Group Representations, 2nd ed., Springer, 1979.

[H-R;II] - - -, Abstract Harmonic Analysis, Volume II, Structure and Analysis on Compact Groups, Analysis on Locally Compact Abelian Groups, 3rd printing, Springer, 1997.

[H] E. Hille, Functional Analysis and Semi-groups, Colloq. Publ., Amer. Math. Soc., 1948.

[H-P] E. Hille and R. S. Phillips, Functional Analysis and Semi-groups, Colloq. Publ., Amer. Math. Soc., 1957.

[Hi] F. Hirsch, Familles résolvantes, générateurs, cogénérateurs, potentiels, Ann. Inst. Fourier (Grenoble) 22 (1972), no. 1, 89-210.

$[\mathrm{J}]$ B. E. Johnson, Centralisers on certain topological algebras, J. London Math. Soc. 39 (1964), 603-614.

[J-K-B] N. L. Johnson, S. Kotz and N. Balakrishnan, Continuous Univariate Distributions, Vol. I, 2nd ed., Wiley, 1994.

[K;I] T. Kato, Remarks on pseudo-resolvents and infinitesimal generators of semi-groups, Proc. Japan Acad. 35 (1959), 467-468.

[K;II] - Perturbation Theory for Linear Operators, Springer, 1966.

[Ki] J. Kisyński, The Widder spaces, representations of the convolution algebra $L^{1}\left(\mathbb{R}^{+}\right)$, and one parameter semigroups of operators, preprint, Inst. Math., Polish Acad. Sci., 1998, 36 pp.

[Kr] S. G. Krein, Linear Differential Equations in Banach Space, Transl. Math. Monographs 29, Amer. Math. Soc., Providence, RI, 1971.

[deL-H-W-W] R. deLaubenfels, Z. Huang, S. Wang and Y. Wang, Laplace transforms of polynomially bounded vector-valued functions and semigroups of operators, Israel J. Math. 98 (1997), 189-207. 
[deL-J] R. deLaubenfels and M. Jazar, Functional calculi, regularized semigroups and integrated semigroups, Studia Math. 132 (1999), 151-172.

[L] J. L. Lions, Les semigroupes distributions, Portugal. Math. 19 (1960), 141-164.

[Pal] T. W. Palmer, Banach Algebras and the General Theory of *-Algebras. Volume I: Algebras and Banach Algebras, Encyclopedia Math. Appl. 49, Cambridge Univ. Press, 1994.

[P] A. Pazy, Semigroups of Linear Operators and Applications to Partial Differential Equations, 2nd printing, Springer, 1983.

[Ph] R. S. Phillips, An inversion formula for Laplace transforms and semigroups of linear operators, Ann. of Math. 59 (1954), 325-356.

[R-Y] D. Revuz and M. Yor, Continuous Martingales and Brownian Motion, Springer, 1991.

[R;I] W. Rudin, Principles of Mathematical Analysis, 3rd ed., McGraw- Hill, 1976.

[R;II] -, Real and Complex Analysis, 2nd ed., McGraw-Hill, 1974.

[S-K] I. E. Segal and R. A. Kunze, Integrals and Operators, 2nd ed., Springer, 1987.

[T] H. F. Trotter, Approximation of semi-groups of operators, Pacific J. Math. 8 (1958), 887-919.

[W;I] D. V. Widder, The Laplace Transform, Princeton Univ. Press, 1946.

[W;II] -, An Introduction to Transform Theory, Academic Press, 1971.

[Y;I] K. Yosida, On the differentiability and the representation of one-parameter semi-groups of linear operators, J. Math. Soc. Japan 1 (1948), $15-21$.

[Y;II] - Functional Analysis, 6th ed., Springer, 1980; reprint of the 1980 edition, Springer, 1995.

Faculty of Electrical Engineering

Technical University of Lublin

Nadbystrzycka 38A, P.O. Box 189

20-618 Lublin, Poland

E-mail: kisynski@antenor.pol.lublin.pl 